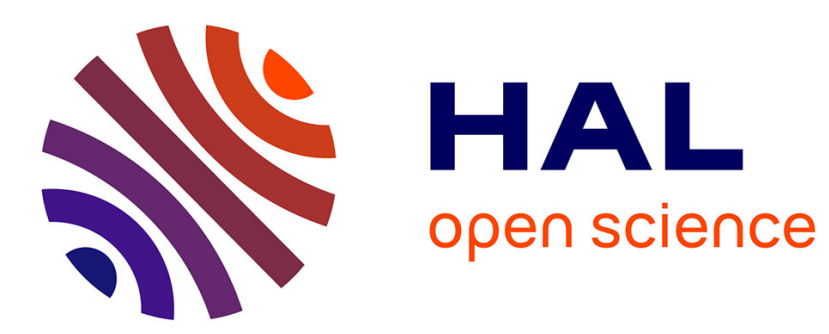

\title{
Morphological modeling of three-phase microstructures of anode layers using SEM images
}

Bassam Abdallah, François Willot, Dominique Jeulin

\section{To cite this version:}

Bassam Abdallah, François Willot, Dominique Jeulin. Morphological modeling of three-phase microstructures of anode layers using SEM images. Journal of Microscopy, 2016, 263 (1), pp.51-63. 10.1111/jmi.12374 . hal-01258887

\section{HAL Id: hal-01258887}

\section{https://hal-mines-paristech.archives-ouvertes.fr/hal-01258887}

Submitted on 19 Jan 2016

HAL is a multi-disciplinary open access archive for the deposit and dissemination of scientific research documents, whether they are published or not. The documents may come from teaching and research institutions in France or abroad, or from public or private research centers.
L'archive ouverte pluridisciplinaire HAL, est destinée au dépôt et à la diffusion de documents scientifiques de niveau recherche, publiés ou non, émanant des établissements d'enseignement et de recherche français ou étrangers, des laboratoires publics ou privés. 


\title{
Morphological modeling of three-phase microstructures of anode layers using SEM images
}

\author{
Bassam Abdallah ${ }^{1}$, François Willot*1 and Dominique Jeulin ${ }^{1}$ \\ ${ }^{1}$ MINES ParisTech, PSL Research university, Centre for mathematical morphology, 35, rue St \\ Honoré, F-77300 Fontainebleau, France
}

\begin{abstract}
A general method is proposed to model 3D microstructures representative of three-phase anode layers used in fuel cells. The models are based on SEM images of cells with varying morphologies. The materials are first characterized using three morphological measurements: (cross-)covariances, granulometry and linear erosion. They are measured on segmented SEM images, for each of the three phases. Second, a generic model for three-phase materials is proposed. The model is based on two independent underlying random sets which are otherwise arbitrary. The validity of this model is verified using the cross-covariance functions of the various phases. In a third step, several types of Boolean random sets and plurigaussian models are considered for the unknown underlying random sets. Overall, good agreement is found between the SEM images and three-phase models based on plurigaussian random sets, for all morphological measurements considered in the present work: covariances, granulometry and linear erosion. The spatial distribution and shapes of the phases produced by the plurigaussian model are visually very close to the real material. Furthermore, the proposed models require no numerical optimization and are straightforward to generate using the covariance functions measured on the SEM images.
\end{abstract}

Keywords: Three-phase random sets; Boolean models; Plurigaussian 3D models; Fuel cells; SOFC

\section{Introduction}

The recent development of fuel cells has been driven by an increasing demand for new energy production, and technological advances (Badwal et al., 2014; Laguna-Bercero, 2012). Since the pioneering work of Grove (1839), fuel cells have been tremendously improved both in terms of performance and durability. The materials involved in the manufacturing process have become complex as per their composition and microstructure. In this regard, electrodes, made of an electrical conductor, an ionic conductor and pores are a key part of solid oxide fuel cells (SOFC). Sophisticated interactions between phases have been reported in commonly-used microstructures (Joos et al., 2012) at the micro- and nano-scale. Thus, the characterization of anode microstructures is a critical point in the improvement of the whole cell. Several works have focused on the reconstruction of a 3D volume using FIB-SEM techniques (Gostovic et al., 2007; Iwai et al., 2010; Joos et al., 2011; Shearing et al., 2009; Wilson et al., 2006). These however require expensive facilities for data acquisition. By contrast, the development of $3 \mathrm{D}$ microstructural models based on $2 \mathrm{D}$ images offers a low-cost easy alternative. Careful study of $2 \mathrm{D}$ images indeed provides estimates of 3D properties such as three-point boundaries (Zhang et al., 2009) but cannot directly provide estimates of geometrical properties such as phases tortuosity, which are useful for estimating the cells's transport properties (Masson et al., 2015). These properties can be measured, however, on 3D models constructed from 2D images, without the need for expensive and time consuming FIB-SEM tomography. Furthermore, the computation of the effective transport properties of the materials, such as ionic diffusion, electronic conductivity and permeability are microstructure-sensitive and require accurate simulations of $3 \mathrm{D}$ microstructures (Abdallah et al., 2015).

The present work is organized as follows. A short presentation of the anode samples is given in Sec. 2.1. The three-phase SEM images are segmented using specific techniques described in Sec. 2.2. The morphology is characterized in terms of various measurements in Secs. 3 and 4. A statistical characterization of the microstructure is provided in Sec. 5. It is shown that the morphological measurements support a strong probabilistic assumption which is especially useful to model the microstructure. Under this assumption, we attempt to model the

${ }^{*}$ Corresponding author. E-mail: francois.willot@mines-paristech.fr, Telephone: +331646948 07, Fax: +33164694707. 


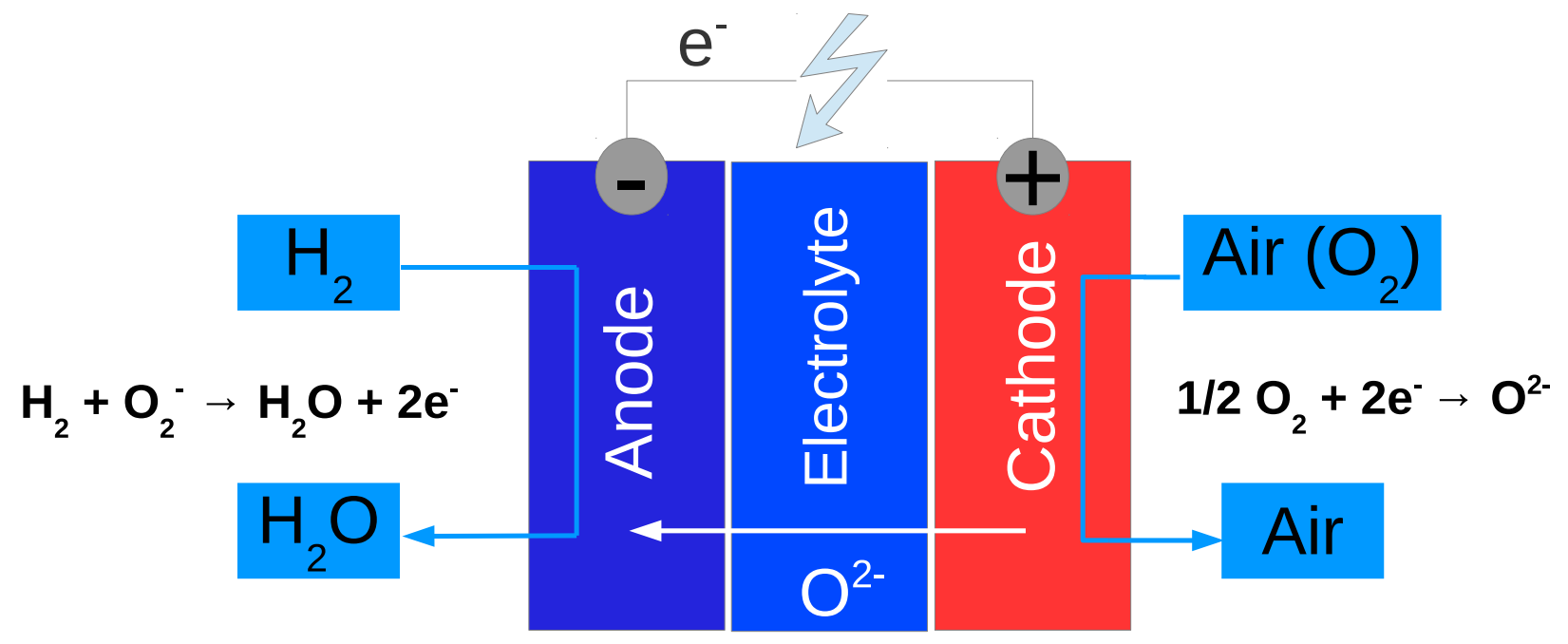

Figure 1: Schematic principle of the fuel cell: Two composite electrode layers separated by a dense electrolyte. At the cathode, di-oxygen molecules (e.g. from air or $\mathrm{H}_{2}$ syngas). are ionised into $\mathrm{O}^{2-}$ anions that go through the dense electrolyte in order to reach the anode side. On the anode compartment $H_{2}$ is injected to react with the oxygen anions and give water plus electrons.

microstructure using Boolean models (Sec. 6) and plurigaussian truncated random functions (Sec. 7). Concluding remarks are in Sec. 8 .

\section{Anode cell layers}

\subsection{Anode samples}

This work is concerned with the microstructural modeling of anode cell layers located at the top and bottom of an electrolyte substrate (see Fig. 1). The layers are heterogeneous materials made of two solid phases, gadoliniumdoped ceria (GDC) and lanthanum-doped strontium titanate (LST), and the pores. Specific transport properties are allowed by each of the three phases. The GDC phase is a molecule with high ionic conduction property (transport of $0^{2-}$ ), LST is an electrical conductor. Fluid flow occurs in the material's porous phase, which results in an effective permeability. The morphology of each of the three phases is constrained by the two others, therefore increasing one of the material's effective properties without degrading the other two is difficult. One minimal requirement is that each of the three phases percolates to allow transport in the material. Additionally, electrochemical properties in anode layers have been found to be especially sensitive to contact surface areas (Yurkiv et al., 2015).

The present work focuses on the morphological modeling of anode cells. The cells were produced at ISTEC (Instituto di Scienza e Tecnologia dei Materiali Ceramici, Italy). Additionally, a few anode cells prepared by the DLR (Deutsches Zentrum für Luft- und Raumfahrt e.V., Germany) and by the Center for Materials (Mines ParisTech, France) are used in the present study for comparison purposes. All cells were analyzed by the Center for Materials (Mines ParisTech, France). To achieve percolation of the two solid phases, the LST and GDC phases were prepared so that both phases have roughly the same volume fraction. The pores volume fraction is not known. The reader is referred to Masson et al. (2015) for details on material preparation. The present study is based on a set of SEM microscopy images of back-scattered electrons representing eight anode cells obtained at Center for Materials (again, the reader is refered to Masson et al. (2015) for details on image acquisition). Each anode cell is symmetrical: the microstructures at the top and bottom of the electrolyte should be very similar. Each of the eight samples have been prepared and processed in different manners. Furthermore, they have been used for varying periods of time. Accordingly, we expect differences in the morphology of each sample. A series of 9 to 14 images of the top (resp. bottom) parts of each sample are used hereafter. Examples of SEM images are shown in Fig. 2. Each SEM image contains $1024 \times 883$ pixels at resolution $12.4 \mathrm{~nm}$ per pixel. A region of interest in the core of each layer, without electrolyte, is selected from each image. The size of the crop varies between $251 \times 1024$ pixels $\left(39.5 \mathrm{\mu m}^{2}\right)$ to $883 \times 1024$ pixels $\left(139 \mathrm{\mu m}^{2}\right)$. Hereafter we denote each ISTEC sample by a number in the range 14 to 32 . The bottom layer is identified with the letter $i$, and the top layer with the letter $s$. For instance, 29-s refers to the top layer of sample 29. 


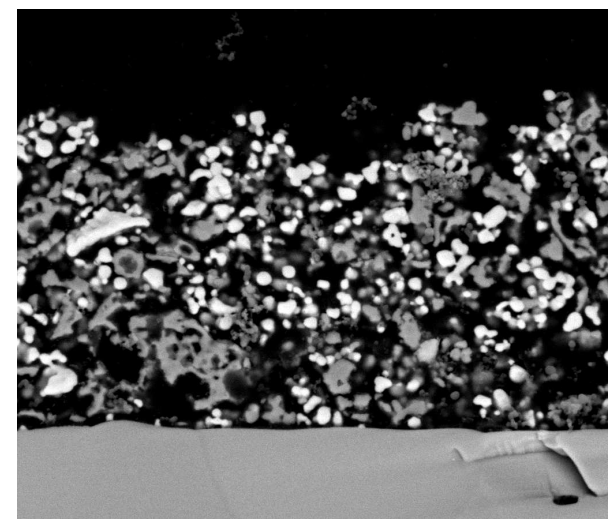

(a)

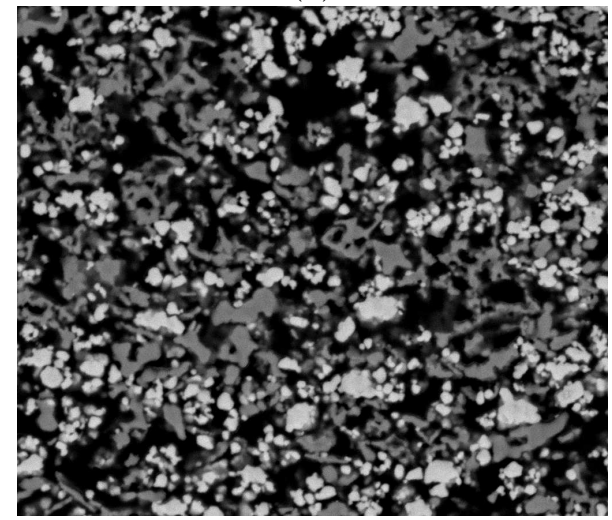

(c)

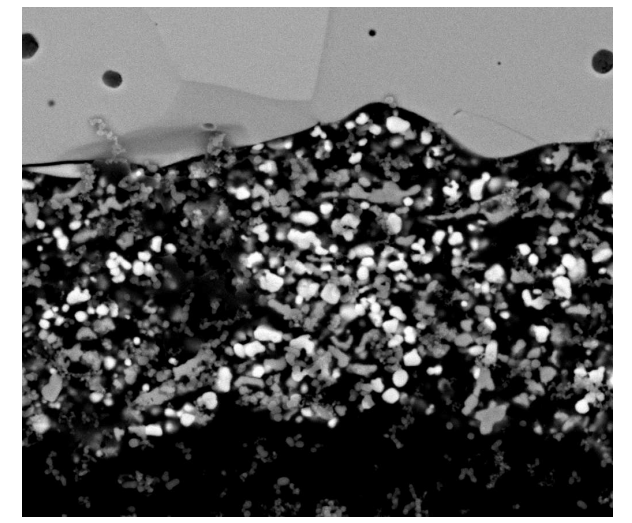

(b)

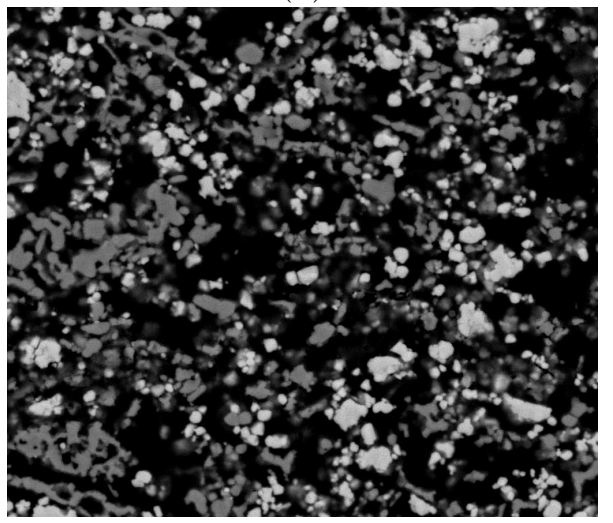

(d)

Figure 2: SEM images of symmetric cells 14 (a; b) and 29 (c; d) manufactured by CNR ISTEC. Images are 1024 pixels $(12.7 \mu \mathrm{m})$ wide and 883 pixels $(11 \mu \mathrm{m})$ height for a spatial resolution of $12.4 \mathrm{~nm} / \mathrm{pixel}$ (images: D. Masson). Images (a) and (c) show the layer on top of the electrolyte, and (b) and (d) on the bottom. Black: pores; white: GDC; dark-gray: LST. The electrolyte is visible in images (a) and (b). 


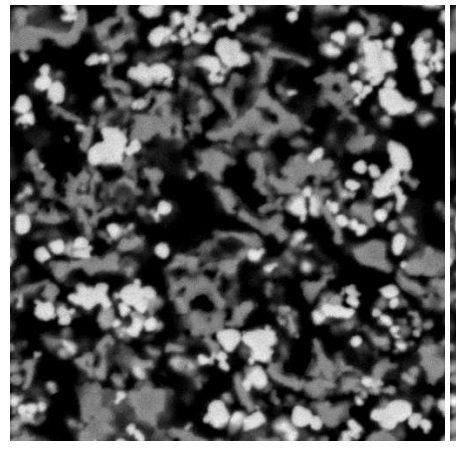

(a)

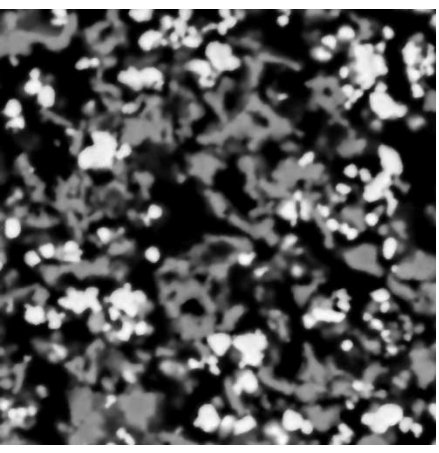

(b)

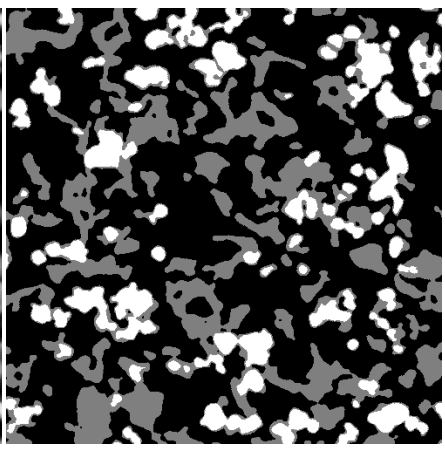

(c)

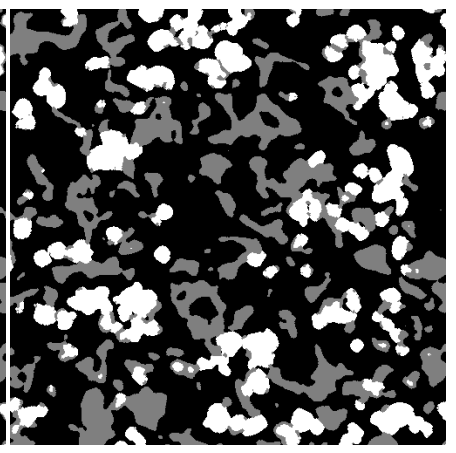

(d)

Figure 3: Segmentations steps of the SEM images illustrated on one enlarged zone in a sample: (a) SEM image; (b) bilateral filtering; thresholding (c); (d) removal of 3D "shadow" effects.

\section{$2.2 \quad$ Image segmentation}

The SEM images are segmented in order to assign each pixel to one of the three phases. The most important steps of the segmentation are illustrated in Fig. (3). We first filter noise using a bilateral filtering (Tomasi and Manduchi, 1998). The latter is applied using the transform:

$$
\mathcal{F}(I ; i, j)=\frac{1}{\mu} \sum_{k, l \in W(i, j)} I(i, j) f\left(|i-k|,|j-l| ; \sigma_{c}\right) f^{\prime}\left(|I(i, j)-I(k, l)| ; \sigma_{i}\right),
$$

where $I$ is the input image, $\mathcal{F}(I)$ its transform, $W(i, j)$ is a square-shaped window of side length $a$ centered on a pixel with coordinates $(i, j), \sigma_{i}$ and $\sigma_{c}$ stand for the standard deviations of the centered Gaussian functions:

$$
f(x, y ; \sigma)=\frac{1}{2 \pi \sigma^{2}} \mathbf{e}^{\frac{-\left(x^{2}+y^{2}\right)}{2 \sigma^{2}}}, \quad f^{\prime}(x, \sigma)=\frac{1}{\sqrt{2 \pi} \sigma} \mathbf{e}^{\frac{-x^{2}}{2 \sigma^{2}}} .
$$

The filter does not remove the contrast at the interface between phases. All images have been acquired on the same microscope, therefore we fix the same set of parameters $\sigma_{i}=0.15, \sigma_{c}=2.5$ and $a=5$ (pixels) for all images. The normalizing parameter $\mu$ reads (Tomasi and Manduchi, 1998):

$$
\mu=\sum_{k, l \in W(i, j)} f\left(|i-k|,|j-l| ; \sigma_{c}\right) f^{\prime}\left(|I(i, j)-I(k, l)| ; \sigma_{i}\right) .
$$

A rough approximation of each phase is obtained by two thresholds on the output $\mathcal{F}(I)$. Noise-reduction is mainly visible when looking at enlargements (Fig. 3b), nevertheless it greatly improves the rest of the segmentation process. In the rest of this study, for simplicity, the GDC, LST and porous phases are denoted respectively by the letter $W$ (white), $G$ (gray) and $B$ (black).

The intensity of the signal in the image results from the scattering of electrons with the material. Therefore, phases containing atoms with the highest atomic number appear brighter than the other phases in the backscattered electron images. As a result, the GDC phase appear in white, the LST phase in gray and the pores in black. However, an unwanted effect should be first taken into account. Due to the halo artifact, the SEM images are not actual planar 2D sections. Parts of the structures under the plane of acquisition are visible in what should really be pores. The latter are located slightly behind the section along which the material was cut. Therefore, we fix the two threshold values that monitor the volume fractions of LST and GDC to minimize the halo effect (Fig. 3c). Note that thresholds are such that the volume fractions of the LST and GDC phases are equal, which leaves one free parameter. After thresholding, a relief effect is still observed around each phase, due to out-of-plane structures. Following Gillibert et al. (2012), a combination of simple mathematical morphology operators are used to remove this effect. We denote by $\delta_{S E}(A)$ and $\gamma_{S E}(A)$ the morphological dilation and opening of a set $A$ by a structural element $S E$. The halo effect is removed by considering the GDC phase $(W)$ and the LST phase $(G)$ and applying the following:

$$
W=W \cup\left(\delta_{S E}(W) \cap\left[G \backslash \gamma_{S E}(G)\right]\right),
$$

with $S E$ taken as a flat-disk of fixed radius. The radius of $S E$ is chosen such as the out-of-plane structures are removed, in this case 3 pixels. This transformation provides the segmented image, such as image (d) in Fig. 3. In this image each pixel is assigned either to the porous phase (black), the LST phase (gray) or the GDC phase (white). 


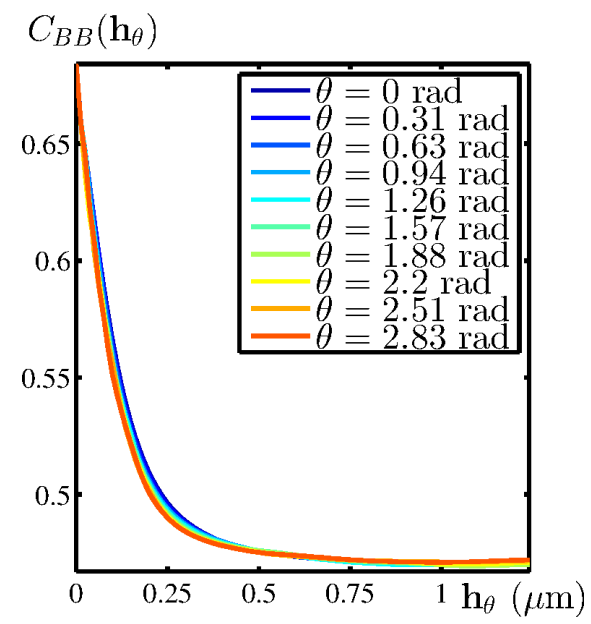

Figure 4: Mean covariance $C_{i i}(\mathbf{h})$ of the porous phase over a sample, as a function of the vector $\mathbf{h}$, parametrized by its norm $h$ and angle with the $x$-axis $\theta$. The angle $\theta$ takes on increasing values $\theta=0, \ldots, \pi$. The isotropy hypothesis is validated since the covariance remains the same regardless the direction of $\mathbf{h}$.

\section{Morphological measurements}

We now use the segmented image to measure various morphological criteria. The images are considered as realizations of a stationary random structure. Therefore all morphological criteria are measured separately on each image, and then averaged on all images that belong to the same sample. We first estimate the covariance and cross-covariance functions (Matheron, 1967) as the probability:

$$
C_{i j}(\mathbf{h})=P\{\mathbf{x} \in i, \mathbf{x}+\mathbf{h} \in j\}
$$

where $i$ and $j \in\{W, G, B\}$ are two phases, and $\mathbf{h}$ is a vector. The values of $\mathbf{x}$ in the above are taken so that both $\mathbf{x}$ and $\mathbf{x}+\mathbf{h}$ are in the observation window. Numerical investigations indicate that the medium is isotropic with respect to the covariance so that $C_{i j}(\mathbf{h})$ depends only on the norm $h=\|\mathbf{h}\|$ of $\mathbf{h}$. This is illustrated on Fig. 4 for the porous phase. Similar results hold for the other phases and all image sets. Accordingly, we identify $C_{i j}(\mathbf{h})$ with $C_{i j}(h)$. Note that the volume fractions of phase $i$ is given by:

$$
f_{i}=P\{\mathbf{x} \in i\}=C_{i i}(0)
$$

We also estimate the following statistical properties:

$$
Q_{i}(\ell)=P\left\{L(\ell) \subset i^{c}\right\}, \quad P_{i}(\ell)=P\{L(\ell) \subset i\}
$$

where $L(\ell)$ is a segment of length $\ell$ with unspecified orientation or location, $i=W, G, B$ is one of the three phases and $i^{c}$ is the complementary set of $i$. In practice, the functions $Q_{i}$ and $P_{i}$ are estimated by computing the area (or volume fraction) of the sets $i$ and $i^{c}$ eroded by segments of length $\ell$. The latter is related to the linear contact distribution. We (abusively) refer to $P_{i}$ and $Q_{i}$ as "linear erosions" hereafter. For general Boolean models with convex grains, linear erosion functions are exponentials (see Sec. 6). This allows one to determine with few computations if a Boolean set might be appropriate to model a material.

The grain size distribution of set $i$ is estimated by the granulometry by opening $G_{i}(S)$. Denoting by $\gamma_{B(S)}(i)$ the morphological opening of the set $i$ by the compact set $B(S)$ of area $S$, one has:

$$
G_{i}(S)=1-P\left\{\mathbf{x} \in \gamma_{B(S)}(i) \mid \mathbf{x} \in i\right\}
$$

In our case $B(S)$ is taken as a disk of surface $S$. This measure is used in Secs. 6 and 7 to validate models.

The representativity of all three-phase models $M$ developed in this work is evaluated quantitatively by the following error criterion $\mathcal{E}(M)$ :

$$
\mathcal{E}(M)=\frac{1}{3} \sum_{i=W, G, B}\left[\left\langle\left|C_{i}(h)-C_{i}^{M}(h)\right|^{2}\right\rangle_{h}^{1 / 2}+\left\langle\left|Q_{i}(\ell)-Q_{i}^{M}(\ell)\right|^{2}\right\rangle_{\ell}^{1 / 2}+\left\langle\left|G_{i}(S)-G_{i}^{M}(S)\right|^{2}\right\rangle_{S}^{1 / 2}\right],
$$

where averages, denoted by $\langle\cdot\rangle$, are taken in the intervals $h<1.5 \mu \mathrm{m}, \ell<1.5 \mu \mathrm{m}$ and $S<1.5 \mu \mathrm{m}^{2}$. 


\begin{tabular}{cccc}
\hline Sample & $f_{W} \pm \epsilon_{f_{W}}$ & $f_{G} \pm \epsilon_{f_{G}}$ & $f_{B} \pm \epsilon_{f_{B}}$ \\
\hline $14-\mathrm{i}$ & $0.151 \pm 0.009$ & $0.203 \pm 0.007$ & $0.646 \pm 0.009$ \\
$14-\mathrm{s}$ & $0.151 \pm 0.009$ & $0.168 \pm 0.008$ & $0.681 \pm 0.009$ \\
$24-\mathrm{i}$ & $0.161 \pm 0.008$ & $0.161 \pm 0.007$ & $0.678 \pm 0.009$ \\
$24-\mathrm{s}$ & $0.179 \pm 0.009$ & $0.198 \pm 0.007$ & $0.624 \pm 0.008$ \\
$25-\mathrm{i}$ & $0.175 \pm 0.009$ & $0.177 \pm 0.007$ & $0.648 \pm 0.009$ \\
$25-\mathrm{s}$ & $0.171 \pm 0.013$ & $0.193 \pm 0.010$ & $0.636 \pm 0.012$ \\
$28-\mathrm{i}$ & $0.185 \pm 0.006$ & $0.196 \pm 0.006$ & $0.619 \pm 0.006$ \\
$28-\mathrm{s}$ & $0.197 \pm 0.005$ & $0.214 \pm 0.005$ & $0.589 \pm 0.005$ \\
$29-\mathrm{i}$ & $0.154 \pm 0.007$ & $0.162 \pm 0.006$ & $0.684 \pm 0.007$ \\
$29-\mathrm{s}$ & $0.187 \pm 0.006$ & $0.207 \pm 0.005$ & $0.605 \pm 0.005$ \\
$32-\mathrm{i}$ & $0.188 \pm 0.009$ & $0.197 \pm 0.008$ & $0.615 \pm 0.009$ \\
\hline
\end{tabular}

Table 1: Volume fractions $f_{i}$ of each phase $i$ estimated on SEM images and their associated relative error $\epsilon_{f_{i}}$.

\section{Representative volume element of the microstructure}

The representativity of the microstructures in terms of volume fraction $f_{i}$ for the phase $i$ is considered. The latter is estimated on $2 \mathrm{D}$ sections by estimating area fractions as explained below.

Consider a $2 \mathrm{D}$ section of area $S_{0}$, either of the real material (SEM images) or of a 3D model $M$. The apparent area fraction of each phase is estimated on disjoint subdomains of surface $S$. The variance of the latter, $D_{f_{i}}^{2}(S)$ scales as shown in (Kanit et al., 2003; Matheron, 1971):

$$
\frac{D_{f_{i}}^{2}(S)}{D_{f_{i}}^{2}} \sim \frac{A_{2}^{f_{i}}}{S}, \quad S \gg A_{2}^{f_{i}},
$$

where $A_{2}^{f_{i}}$ is the integral range and $D_{f_{i}}^{2}$ is the point variance for phase $i$, i.e.:

$$
D_{f_{i}}^{2}=f_{i}\left(1-f_{i}\right)
$$

The integral range $A_{2}^{f_{i}}$ is the integral of the correlation function of phase $i$; we refer to Matheron (1971) for a discussion of its main properties. Simply remark that when (9) holds, the domain $S$ acts as $n=S / A_{2}^{f_{i}}$ independent sub-domains each of area $A_{2}^{f_{i}}$. Eq. (9) is useful to estimate the representativity of a microstructure. For instance, for a given domain of area $S$ the relative error $\epsilon_{f_{i}}$ on the volume fraction $f_{i}$ estimated for phase $i$ is given by:

$$
\epsilon_{f_{i}}=\frac{2 D_{f_{i}}}{f_{i}} \sqrt{\frac{A_{2}^{f_{i}}}{S}}
$$

In the next sections, the function $D_{f_{i}}^{2}(S)$ is used to compare simulations of the models and the SEM images.

Tab. 1 gives estimates of the area fractions, and therefore the volume fractions $f_{i}$ of each phase, averaged over the 11 samples. The porosity is around $f_{B} \approx 63 \%$ for all samples, while the volume fraction of GDC and LST lies between $15 \% \leq f_{G, W} \leq 20 \%$. The integral range $A_{2}^{f_{i}}$ is extracted numerically by fitting the left-hand side of (9) for large S. This value is used to estimate $\epsilon_{f_{i}}$ in Eq. 11. As seen in Tab. 1, the volume fractions of the two solid phases $f_{G}$ and $f_{W}$ differ by up to $10 \%$, except for sample 14 -i for which the relative difference is $25 \%$. These differences are much higher than the relative errors $\epsilon_{f_{i}}$, and are presumably an effect of the preparation techniques.

\section{Three-phase media modelled using two underlying indepen- dent random sets}

In this section, we assume that the three sets $B, W$ and $G$ are obtained from two independent random sets $X$ and $Y$. To obtain three random sets $A_{i}(i=1,2,3)$, we consider the following combinations of the two sets $X$ and $Y$ :

$$
A_{1}=X, \quad A_{2}=X^{c} \cap Y, \quad A_{3}=X^{c} \cap Y^{c} .
$$

Each set $A_{i}(i=1,2,3)$ is used to represent either $B, W$ or $G$. The above construction is represented schematically in Fig. 5. We denote by $C_{i}(h)$ and $C_{i j}(h)(i, j=1,2,3)$ the covariance and cross-covariance, respectively, of the sets $A_{i}$ and $A_{j}$ as in (4). The covariance of the complementary set $A_{i}^{c}$, denoted by $\bar{C}_{i}(h)$, is given by:

$$
\bar{C}_{i}(h)=1-2 C_{i}(0)+C_{i}(h)
$$




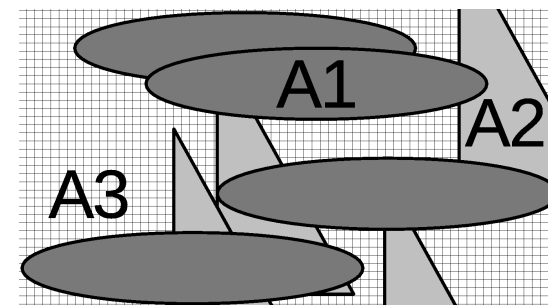

Figure 5: Schematic representation of a three-phase material $A_{1}$ (dark gray), $A_{2}$ (light gray), $A_{3}$ (crosshatch) modeled by two underlying independent random sets $X$ (ellipses) and $Y$ (triangles).

The covariances $C_{i}(h)$ of the sets $A_{i}$ read:

$$
C_{1}(h)=C_{X}(h), \quad C_{2}(h)=\bar{C}_{X}(h) C_{Y}(h), \quad C_{3}(h)=\bar{C}_{X}(h) \bar{C}_{Y}(h),
$$

where $C_{X}(h)$ and $C_{Y}(h)$ are the covariances of the sets $X$ and $Y$ respectively, and $\bar{C}_{X}(h)$ and $\bar{C}_{Y}(h)$ that of their complementary sets $X^{c}$ and $Y^{c}$. The independency of $X$ and $Y$ implies, for the cross-covariance functions $C_{i j}(h)($ Jeulin , 2014):

$$
\begin{aligned}
C_{12}(h) & =\frac{C_{2}(0)}{1-C_{1}(0)}\left[C_{1}(0)-C_{1}(h)\right], \\
C_{13}(h) & =\frac{C_{3}(0)}{1-C_{1}(0)}\left[C_{1}(0)-C_{1}(h)\right], \\
C_{23}(h) & =\frac{C_{2}(0)}{1-C_{1}(0)} \overline{C_{1}}(h)-C_{2}(h) .
\end{aligned}
$$

For instance, formula (15) is obtained as follows:

$$
\begin{aligned}
C_{12}(h) & =P\left\{\mathbf{x} \in A_{1}, \mathbf{x}+\mathbf{h} \in A_{2}\right\} \\
& =P\left\{\mathbf{x} \in X, \mathbf{x}+\mathbf{h} \in X^{c} \cap Y\right\} \\
& =P\left\{\mathbf{x} \in X, \mathbf{x}+\mathbf{h} \in X^{c}\right\} P\{\mathbf{x}+\mathbf{h} \in Y\} \\
& =\left[C_{X}(0)-C_{X}(h)\right] C_{Y}(0) .
\end{aligned}
$$

Eqs. (16) and (17) are derived in a similar way. This particular structure, resulting from the construction of the model, is useful to test the validity of the independence assumptions from experimental covariances. We emphasize that (15), (16) and (17) are valid if $X$ and $Y$ are independent. This assumption is tested by considering all possible choices for assigning any of the sets $B, G$ or $W$ to $A_{1}, A_{2}$ or $A_{3}$. The 6 possibilities, labelled $H_{k}(1 \leq k \leq 6)$ are given in Tab. 2. We denote $C_{i j}^{H_{k}}(h)$ where $(i, j) \in\{B, G, W\}$ the cross-covariance $C_{i j}$ defined in (15), (16) and (17) with the $A_{i}$ specified by $H_{k}(1 \leq k \leq 6)$.

For each pair of values $i \neq j$ and each of the possible set of choices $H_{k}(1 \leq k \leq 6)$, the cross-covariance $C_{i j}^{H_{k}}(h)$ is computed according to (15), (16), (17) and compared to its estimate directly measured on the SEM image. For instance, referring to Tab. 2, $C_{12}^{H_{1}}(h)$ is the cross-covariance between sets B and G, which is compared to the right-hand-side of Eq. (15). The latter is computed using the covariance of the sets B and the volume fraction of the set G. Accordingly, hypothesis $H_{1}$ implies that:

$$
C_{G B}(h), C_{G B}^{H_{1}}(h)=\frac{C_{G}(0)}{1-C_{B}(0)}\left[C_{B}(0)-C_{B}(h)\right]
$$

are equal. The cross-covariances $C_{G W}^{H_{k}}$ of $G$ and $W$ are plotted in Fig. 6 for sample 29-i, for all hypothesis $H_{1}, \ldots$, $H_{6}$, and compared to $C_{G W}$.

In order to quantify the quality of each possible choice $H_{k}$ we compute the relative error:

$$
E(k)=\sum_{i, j} \frac{1}{n+1} \sum_{h=1}^{n} \frac{\left|C_{i j}^{H_{k}}(h)-C_{i j}(h)\right|}{C_{i j}(h)}
$$

for all image sets and set of choices $H_{k}$ (Tab. 3).

The configuration $H_{6}$ minimizes the relative error $\varepsilon_{k}$ on 12 out of 16 image sets. Furthermore, $E(6)$ is close to the minimum value on 3 out of the 4 other samples. In the following, we assume that hypothesis $H_{6}$ holds, i.e. 


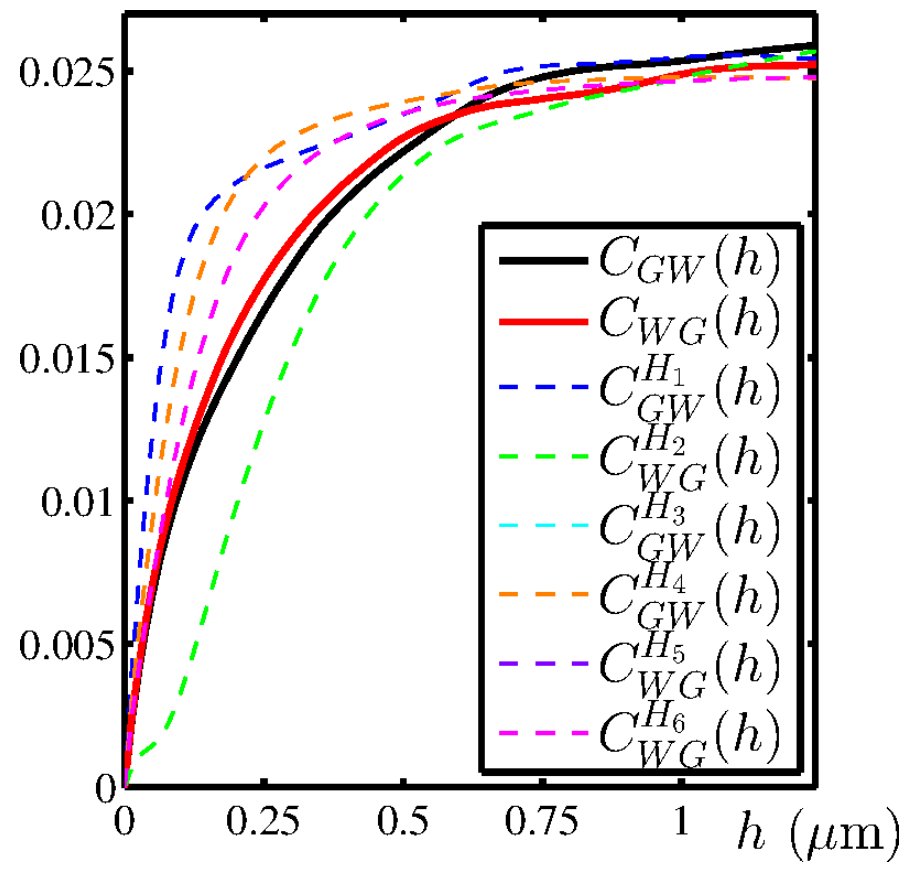

Figure 6: Cross-covariance $C_{G W}$ between sets $G$ and $W$ measured on images of sample 29-s (solid line). Comparison with the cross-covariances $C_{G W}^{H_{k}}$ computed using (15), (16) and (17). The latter are computed for each configuration $k=1, \ldots, 6$ from Tab. (2).

\begin{tabular}{cccc}
\hline Hypothesis & $A_{1}=X$ & $A_{2}=X^{c} \cap Y$ & $A_{3}=X^{c} \cap Y^{c}$ \\
\hline$H_{1}$ & $B$ & $G$ & $W$ \\
$H_{2}$ & $B$ & $W$ & $G$ \\
$H_{3}$ & $G$ & $B$ & $W$ \\
$H_{4}$ & $G$ & $W$ & $B$ \\
$H_{5}$ & $W$ & $B$ & $G$ \\
$H_{6}$ & $W$ & $G$ & $B$ \\
\hline
\end{tabular}

Table 2: The 6 possible choices for assigning any of the sets $B, G$ or $W$ to $A_{1}, A_{2}$ or $A_{3}$.

the three phases are written as (12) with $A_{1}=W, A_{2}=G$ and $A_{3}=B$ as specified in Tab. 2. In particular, using the independency of $X$ with respect to $Y$ :

$$
f_{W}=f_{X}, \quad f_{G}=\left(1-f_{X}\right) f_{Y} .
$$

These equations give the volume fractions of $X$ and $Y$ for each sample (Tab. 1).

Herefter, we aim to determine the random sets $X$ and $Y$, which can be generated independently from each other. Boolean (Sec. 6) and plurigaussian models (Sec. 7) are explored in the following.

\section{Boolean models}

The Boolean model is a set $A \subset \mathbb{R}^{3}$ obtained by implantation of the primary grains $A^{\prime}$ (with possible overlaps) on points $\mathbf{x}_{k}$ distributed according to a Poisson point process $\mathbb{P}$ with intensity $\theta$ (Matheron, 1967):

$$
A=\bigcup_{x_{k} \in \mathbb{P}} A_{\mathbf{x}_{k}}^{\prime},
$$

where $A_{x_{k}}^{\prime}=\left\{\mathbf{x}+\mathbf{x}_{k} ; \mathbf{x} \in A^{\prime}\right\}$ is the translation of $A^{\prime}$ by $\mathbf{x}_{k}$. To determine if the set $X$ or $Y$ might be approached by Boolean models, we measure the linear erosion $Q_{i}(\ell)$ (see 6) for the three phases $G, W$ and $B$ on all data sets. Results are represented in Fig. 7 in lin-log scale for sample 29-s and show an exponential decrease $\log \left[Q_{i}(\ell)\right] \sim \ell$. A similar behavior is observed on all samples. This scaling law is compatible with a Boolean model with convex 


\begin{tabular}{ccccccc}
\hline Image set & $H_{1}$ & $H_{2}$ & $H_{3}$ & $H_{4}$ & $H_{5}$ & $H_{6}$ \\
\hline $14-\mathrm{i}$ & 3.15 & 3.15 & 2.9 & 2.9 & 14.11 & 1.4 \\
$14-\mathrm{s}$ & 4.14 & 3.99 & 3.84 & 3.86 & 6.55 & 2.07 \\
$15-\mathrm{i}$ & 3.61 & 3.42 & 3.2 & 3.21 & 17.06 & 4.19 \\
$15-\mathrm{s}$ & 3.94 & 3.97 & 2.52 & 2.52 & 19.46 & 1.96 \\
$24-\mathrm{i}$ & 4.24 & 4.31 & 3.27 & 3.27 & 3.88 & 2.76 \\
$24-\mathrm{s}$ & 3.62 & 3.84 & 3.33 & 3.31 & 7.64 & 2.38 \\
$25-\mathrm{i}$ & 5.9 & 5.83 & 3.53 & 3.53 & 4.05 & 1.76 \\
$25-\mathrm{s}$ & 4.96 & 4.84 & 4.34 & 4.35 & 7.45 & 1.87 \\
$28-\mathrm{i}$ & 3.23 & 3.26 & 3.96 & 3.96 & 5.18 & 2.44 \\
$28-\mathrm{s}$ & 2.18 & 2.16 & 3.74 & 3.74 & 5.99 & 2.43 \\
$29-\mathrm{i}$ & 3.95 & 3.82 & 3.48 & 3.48 & 4.62 & 1.85 \\
$29-\mathrm{s}$ & 3.17 & 3.16 & 3.96 & 3.96 & 7.16 & 2.42 \\
$32-\mathrm{i}$ & 3.21 & 3.17 & 2.97 & 2.98 & 4.39 & 1.77 \\
$32-\mathrm{s}$ & 3.28 & 3.31 & 3.64 & 3.63 & 7.57 & 3.43 \\
$33-\mathrm{i}$ & 2.94 & 2.88 & 4.16 & 4.16 & 5.86 & 3.45 \\
$33-\mathrm{s}$ & 2.75 & 2.71 & 2.97 & 2.97 & 4.15 & 2.17 \\
\hline
\end{tabular}

Table 3: Relative error $E(k)$ (Eq. (18)) between the measured cross-covariances $C_{i j}(h)$ and the ones computed using (15), (16) and (17) for all combinations $H_{1}, \ldots, H_{6}$, denoted $C_{i j}^{H_{k}}(h)$. For each sample, the minimum value of $E(k)$, corresponding to the best configuration from Tab. 2, is highlighted in grey.

primary grain $A^{\prime}$. Indeed, the covariance $\bar{C}_{A}(h)$ and linear erosion $Q_{A}(\ell)$ of a Boolean model $A$ read (Matheron, 1967):

$$
\bar{C}_{A}(h)=q^{2-r_{A^{\prime}}(h)}, \quad Q_{A}(\ell)=q^{1-\ell r_{A^{\prime}}^{\prime}(0)},
$$

where $q$ is the volume fraction of the complementary set of $A, r_{A^{\prime}}(h)$ is the normalized geometrical covariogram of the primary grain $A^{\prime}$ and $r_{A^{\prime}}^{\prime}(h)$ its derivative. The latter is given by Matheron (1967) in three dimensions:

$$
r_{A^{\prime}}(h)=\frac{\mu_{3}\left(A^{\prime} \cap A_{-h}^{\prime}\right)}{\mu_{3}\left(A^{\prime}\right)},
$$

where $\mu_{3}$ is the Lebesgue measure in dimension 3. Note that the exponential scaling law is expected to hold for the linear erosions of the underlying random sets $X$ and $Y$. Indeed:

$$
Q_{X}(\ell)=Q_{W}(\ell), \quad P_{B}(\ell)=Q_{X}(\ell) Q_{Y}(\ell) .
$$

The above suggests that one uses Boolean random sets to model $X$ and $Y$. We emphasize that the exponential decrease holds for general isotropic Boolean models built on a distribution of convex random primary grains $A^{\prime}$ with arbitrary shape and orientations. Accordingly, in the following, we consider various primary grains $A^{\prime}$ oriented uniformly on the unit sphere. The two models $X$ and $Y$ are determined by their primary grains $A_{X}^{\prime}$ and $A_{Y}^{\prime}$, respectively, and by the Poisson intensity $\Phi_{X}$ and $\Phi_{Y}$. In (Greco, 1979), such a combination of Boolean models with parallelipiped primary grains was used to model iron ore sinter textures.

The round-shaped GDC particles and the elongated shapes observed for the LST phase suggest that one uses spheres of radius $R_{X}$ for $A_{X}^{\prime}$ and cylinders of radius $R_{Y}$ and height $L_{Y}$ for $A_{Y}^{\prime}$. Taking into account the Poisson intensities $\Phi_{X}$ and $\Phi_{Y}$, the entire model, denoted $M_{1}$, depends on five parameters. The normalized geometrical covariogram reads, for the spherical primary grain $A_{X}^{\prime}$ :

$$
r_{X}(h)=1-\frac{3 h}{4 R_{X}}+\frac{h^{3}}{16\left(R_{X}\right)^{3}}, \quad h \leq 2 R_{X}
$$

The normalized mean covariogram of cylinders takes the form of a one-dimensional integral which can be solved analytically using special functions Willot (2015). The covariograms of spheres and cylinders allow to compute the covariance $C_{B, G, W}(h)$ of $B, G$ and $W$ using (13), (14), and (21) without generating 3D Boolean models. The quadratic difference between the covariances of the model and that measured on the images is used as error criterion.

We minimize numerically the error criterion by optimizing the 5 parameters of the model using the algorithm of Nelder and Mead (1965). The optimal microstructure for model $M_{1}$ is found for $R_{X}^{M_{1}}=14 \mu \mathrm{m}, \Phi_{X}^{M_{1}}=$ $1.3110^{-5} \mu^{-3}, R_{Y}^{M_{1}}=8 \mu \mathrm{m}, L_{Y}^{M_{1}}=147 \mu \mathrm{m}$ and $\Phi_{Y}^{M_{1}}=6.7010^{-6} \mu^{-3}$ for sample 29-s. Accurate volume fractions are recovered for the three phases: $f_{B}=0.71 \%, f_{G}=16 \%, f_{W}=15 \%(\mathrm{SEM})$ compared to $\widetilde{f_{B}}=68 \%, \widetilde{f_{G}}=16 \%$ 


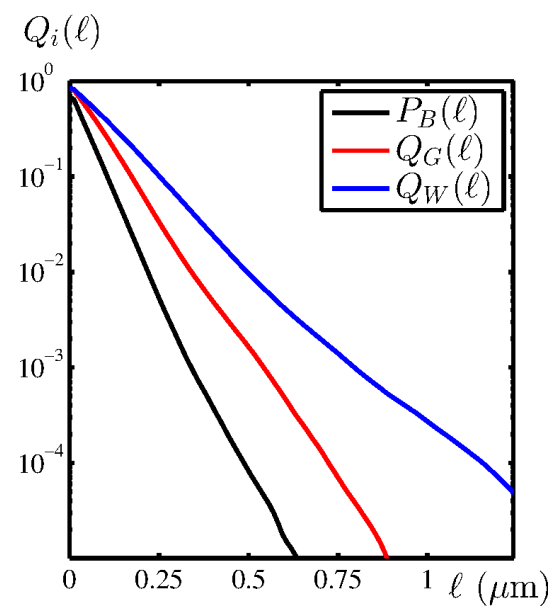

Figure 7: Linear erosion function $Q_{i}(\ell)$ of each phase of the composite 29-s, averaged over all 8 images of the sample and over 10 directions, represented in lin-log scale. The exponential decrease is compatible with a Boolean model with convex grains (see Eq. 21)

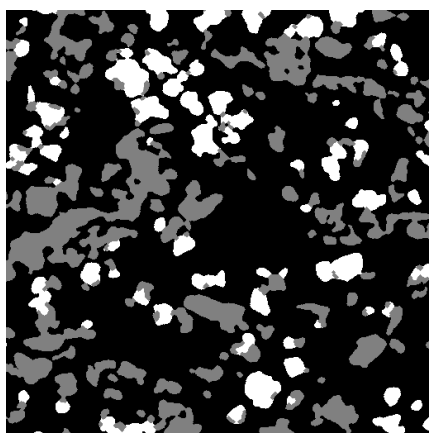

(a)

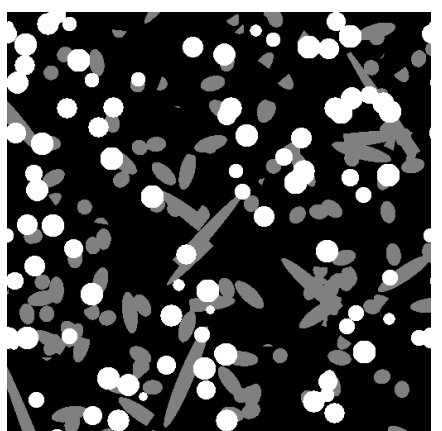

(b)

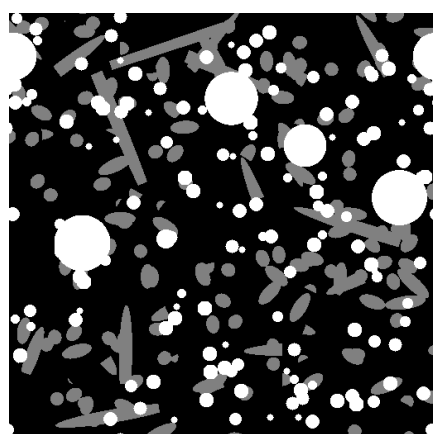

(c)

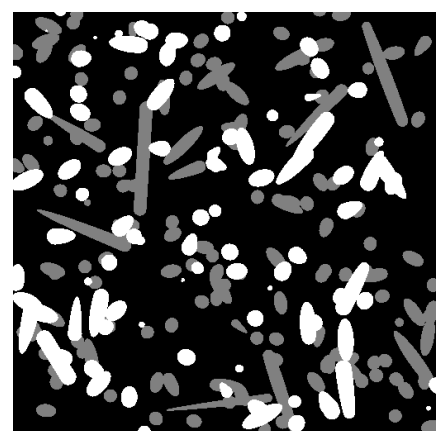

(d)

Figure 8: Sample 29-s: crop of a segmented image (a) and 2D sections of various optimized Boolean models (b-d): model $M_{1}$ with mono-sized spheres for $X$ and cylinders for $Y$ (b), model $M_{2}$ with two populations of spheres for $X$ (c) and model $M_{3}$ made of sphero-cylinders for $X$ and $Y$ (d)

and $\widetilde{f_{W}}=14 \%$ (model). Good agreement is found as well for the covariance functions and linear erosions (Fig. 9a and $9 \mathrm{~b}$ ). However, the model is unable to reproduce the granulometries measured on the SEM images (Fig. 9c), which is much wider than the narrow distribution of sizes obtained with the model. The model is not visually satisfactory either. A $2 \mathrm{D}$ section of $M_{1}$ is represented in Fig. 8b, which is quite different from the SEM images (Fig. 8a).

Two other Boolean models are explored as an attempt to obtain wider granulometries for phases $\mathrm{G}$ and $\mathrm{W}$. In the first one, denoted $M_{2}$, the set $X$ is a Boolean model containing two populations of spheres. The spheres have radius $R_{X}^{M_{2}}$ with probability $s^{M_{2}}$ and $R_{X}^{\prime M_{2}}$ with probability $1-s^{M_{2}}$. The set $Y$, a Boolean model of cylinders, is left unchanged. The model $M_{2}$ is accordingly parametrized by 7 variables: the Poisson intensities $\Phi_{X, Y}^{M_{2}}$ of $X$ and $Y$, the spheres radii $R_{X}^{M_{2}}$ and $R_{X}^{M_{2}}$, the probability $s^{M_{2}}$, the radius $R_{Y}^{M_{2}}$ and length $L_{Y}^{M_{2}}$ of the primary grains in model $Y$. In the second model, denoted $M_{3}$, the two sets $X$ and $Y$ are Boolean models of sphero-cylinders. This model is parametrized by 6 variables: the intensities of the Poisson point processes $\Phi_{X, Y}^{M_{3}}$ and the lengths $L_{X, Y}^{M_{3}}$ and radii $R_{X, Y}^{M_{3}}$ of the sphero-cylinders. Sphero-cylinders are used in order to reproduce the smooth boundaries observed on the segmented image.

The parameters of models $M_{2}$ and $M_{3}$ are optimized, as before, on the covariances of the three phases. Again, the quadratic difference between covariances measured on the segmented images and computed on the models is used as error criterion. A different optimization is carried out for each sample. Using (21), the optimization of model $M_{2}$ is carried out without generating the microstructures. Again, the algorithm of Nelder \& Mead is used. For model $M_{2}$, the optimal microstructure is found when $\Phi_{X}^{M_{2}}=1.8410^{-7} \mu^{-3}, D_{X}^{M_{2}}=75 \mu \mathrm{m}, \Phi_{X}^{\prime M_{2}}=$ 


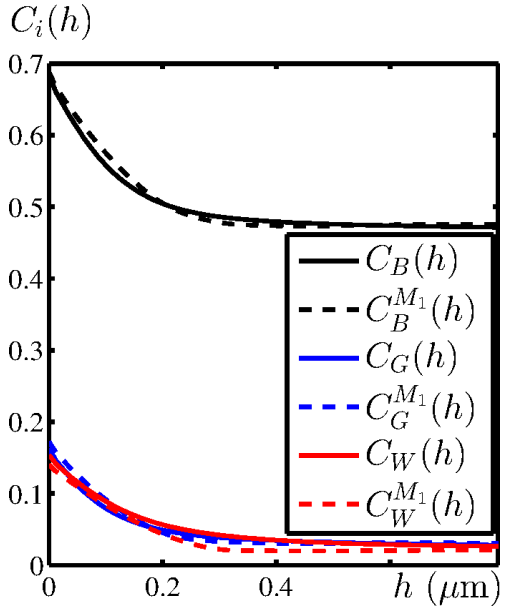

(a)

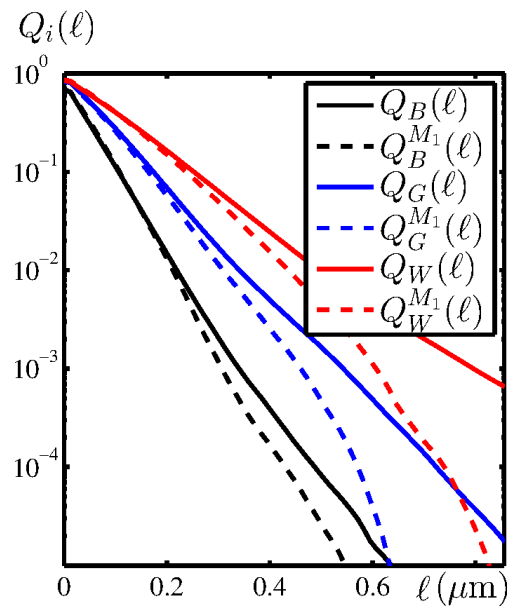

(b)

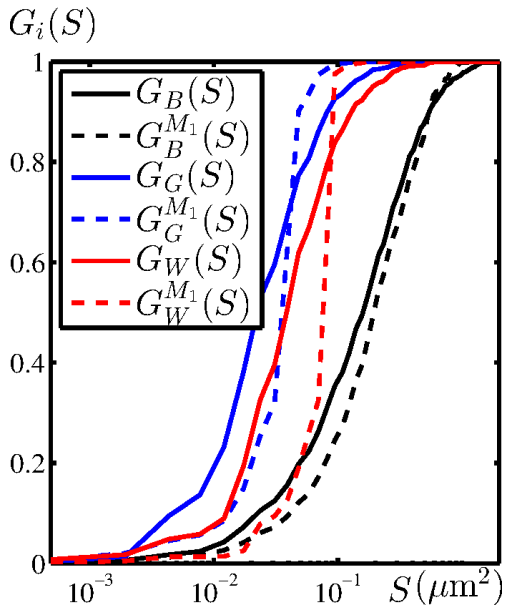

(c)

Figure 9: Sample 29-s: covariances $C_{i}(h)(\mathrm{a})$, linear erosion $Q_{i}(\ell)(\mathrm{b})$ and granulometries $G_{i}(h)(\mathrm{c})$ of the segmented SEM image (solid lines) and that of the optimal Boolean model $M_{1}$ of mono-sized spheres and cylinders (dotted lines) for phases B (black), G (blue) and W (red).

$2.4210^{-5} \mathrm{\mu m}^{-3}, D_{X}^{\prime M_{2}}=21 \mu \mathrm{m}, \Phi_{Y}^{M_{2}}=2.0410^{-5} \mu \mathrm{m}^{-3}, D_{Y}^{M_{2}}=11 \mu \mathrm{m}, L_{Y}^{M_{2}}=113 \mu \mathrm{m}$ for sample 29-s. For model $M_{3}$ we find $R_{X}^{M_{3}}=8 \mu \mathrm{m}, R_{Y}^{M_{3}}=10 \mu \mathrm{m}, L_{X}^{M_{3}}=123 \mu \mathrm{m}, L_{Y}^{M_{3}}=75 \mu \mathrm{m}, \Phi_{X}^{M_{3}}=8.010^{-6} \mu \mathrm{m}^{-3}$ and $\Phi_{Y}=$ $8.210^{-6} \mathrm{\mu m}^{-3}$ for the same sample. 2D sections of the optimized models $M_{2}$ and $M_{3}$ are represented in Fig. 8c and Fig. 8d respectively. The models are not visually satisfactory. Quantitatively, only a small improvement on the granulometry is observed when using model $M_{2}$, compared to model $M_{1}$ (not shown). Similar results are obtained for model $M_{3}$ (not shown).

Finally, we mention that we considered a fourth model that consists of a Boolean model of spheres for set $X$, with radii uniformly distributed from 0 to a maximum value, and a Boolean model of cylinders for set $Y$. Again however, the models were unable to reproduce the observed granulometry.

\section{Plurigaussian model}

In this section, we model sets $X$ and $Y$ by means of truncated Gaussian models (Armstrong et al., 2011; Lantuéjoul, 2002). This choice is led by the need to generate three-phase microstructures with smooth boundaries. Such models have been used successfully to represent food microstructures (Bron and Jeulin, 2004) and (binary) SOFC electrodes (Lanzini et al., 2009).

The three-phase microstructures are represented by two underlying independent sets $X$ and $Y$, as described in Sec. 5. The latter sets are both generated by the convolution of a Gaussian noise $U$ with a weight function $w$. The resulting fields are thresholded. The value of the threshold is chosen to reproduce the measured volume fractions of the three phases. For set $X$, for instance, we use:

$$
\begin{aligned}
X & =\left\{\mathbf{x} \in \Omega ; Z_{X}(\mathbf{x}) \geq \lambda_{X}\right\} \\
Z_{X}(\mathbf{x}) & =\left(w_{X} * U_{X}\right)(\mathbf{x}), \quad U_{X}(\mathbf{x}) \sim \mathcal{N}(0,1),
\end{aligned}
$$

where $*$ is the convolution product, $\lambda_{X}$ is the threshold and $U_{X}(\mathbf{x})$ follows the normal distribution $\mathcal{N}(0,1)$ of mean 0 and variance 1 . The expectation of the indicator function of the random set $X$ reads:

$$
f_{X}=P\left\{Z_{X}(x) \geq \lambda_{X}\right\}=P\left\{\mathcal{N}(0,1) \geq \lambda_{X}\right\},
$$

$P\left\{\mathcal{N}(0,1) \geq \lambda_{X}\right\}$ is the probability that a variable following the normal distribution is greater than $\lambda_{X}$. This equation provides the value for the threshold $\lambda_{X}$. Indeed:

$$
\lambda_{X}=F^{-1}\left(1-f_{X}\right),
$$

where $F$ is the cumulative distribution function of the normal distribution and the volume fraction $f_{X}$ is given by (19) and by the measurements on segmented SEM images (Tab. 1). The weight function $w_{X}$ is normalized and symmetric:

$$
\int_{\Omega} w_{X}^{2}(\mathbf{x}) \mathrm{d} \mathbf{x}=1, \quad w_{X}(\mathbf{x})=w_{X}(-\mathbf{x}), \quad \mathbf{x} \in \Omega .
$$




\begin{tabular}{cccccc}
\hline Sample & $\mathcal{E}\left(M_{1}\right)$ & $\mathcal{E}\left(M_{2}\right)$ & $\mathcal{E}\left(M_{4}\right)$ & $\mathcal{E}\left(M_{5}\right)$ & $\mathcal{E}\left(M_{6}\right)$ \\
\cline { 1 - 5 } $14-\mathrm{i}$ & 4.62 & 6.73 & 6.08 & 5.71 & 7.47 \\
$14-\mathrm{s}$ & 4.94 & 5.88 & 4.66 & 5.43 & 5.16 \\
$24-\mathrm{i}$ & 5.23 & 7.03 & 5.13 & 5.31 & 4.40 \\
$24-\mathrm{s}$ & 4.39 & 5.85 & 4.50 & 4.63 & 3.03 \\
$25-\mathrm{i}$ & 5.52 & 7.40 & 8.58 & 4.59 & 7.56 \\
$25-\mathrm{s}$ & 6.27 & 7.72 & 5.78 & 4.68 & 7.69 \\
$28-\mathrm{i}$ & 5.26 & 6.94 & 4.39 & 5.47 & 3.79 \\
$28-\mathrm{s}$ & 5.75 & 8.23 & 3.56 & 4.31 & 3.97 \\
$29-\mathrm{i}$ & 5.25 & 6.58 & 4.10 & 5.40 & 2.44 \\
$29-\mathrm{s}$ & 5.02 & 6.97 & 3.57 & 4.38 & 2.89 \\
$32-\mathrm{i}$ & 6.28 & 8.97 & 6.06 & 6.25 & 5.81 \\
\hline
\end{tabular}

Table 4: Global error $\left(\times 10^{-3}\right) \mathcal{E}(M)$ (see Eq. 8) computed on models $M_{1-2}$ and $M_{4-6}$, for 11 samples. The best fit obtained for each sample is highlighted in grey.

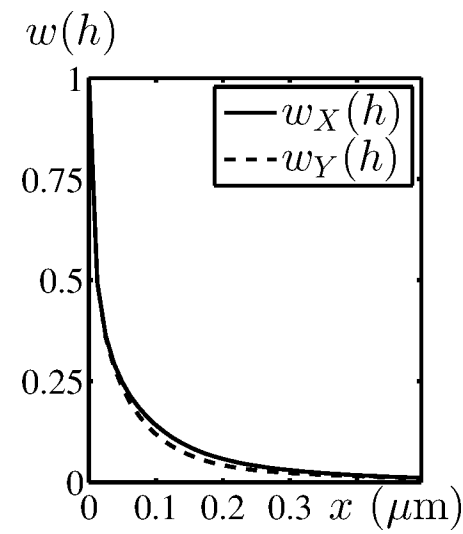

Figure 10: Weight functions $w_{X}$ and $w_{y}$ for sample 29-i. Each weight function is defined by (30) where $\rho_{X, Y}$ is obtained by numerical inversion of (29).

The latter is directly related to the covariance $C_{X}(h)$ of $X$ by (Lantuéjoul, 2002):

$$
C_{X}(h)=\frac{1}{2 \pi} \int_{0}^{\rho_{X}(h)} \frac{1}{\sqrt{1-t^{2}}} e^{\frac{-\lambda_{X}^{2}}{1+t}} \mathrm{~d} t,
$$

where $\rho_{X}(h)=\left(w_{X} * w_{X}\right)(h)$. The function $w_{X}$ is obtained using:

$$
w_{X}=F F T^{-1}\left\{\sqrt{F F T\left\{\rho_{X}\right\}}\right\},
$$

where FFT and $\mathrm{FFT}^{-1}$ are the forward and backward discrete fast Fourier transforms. Once $\lambda_{X}$ is known, the function $\rho$ is provided by inverting numerically (29) and $w_{X}$ is given by (30). We emphasize that the covariance measured on the SEM image for the set $X$ (i.e., $W$ ) is reproduced exactly by the model.

The same procedure is applied to determine $Y$. Recall that, under assumption $H_{6}, W=X, G=X^{c} \cap Y$ and $B=X^{c} \cap Y^{c}$. Accordingly, the covariance of the set $X$ is given by that of $W$ whereas the covariance of $Y$ (see Eq. 14) reads:

$$
C_{X}(h)=C_{W}(h), \quad C_{Y}(h)=\frac{C_{G}(h)}{\bar{C}_{W}(h)} .
$$

As an example, the weight functions $w_{X}$ and $w_{Y}$ corresponding to $X$ and $Y$ are plotted in Fig. 10.

A microstructure model of size $512^{3}$ voxels $\left(6.14 \mu^{3}\right)$ is simulated for each of the 16 samples. A $2 \mathrm{D}$ section of the resulting model is represented in Fig. 13 and compared to the segmented image for sample 29-s. The covariance functions, linear erosions and cumulative granulometries of the three phases are estimated on realizations of the plurigaussian model. For a given model, 10 random 2D sections are selected. The morphological measurements are averaged over the $2 \mathrm{D}$ sections.

The plurigaussian model defined above is hereafter denoted $M_{4}$. The contours of this model, represented in Fig. 11b, are very noisy, as previously observed by Bron and Jeulin (2004). In (Bron and Jeulin, 2004), noise is 


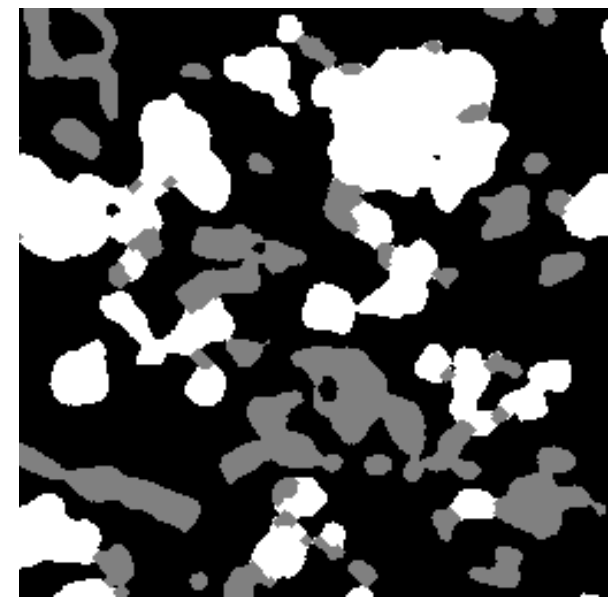

(a)

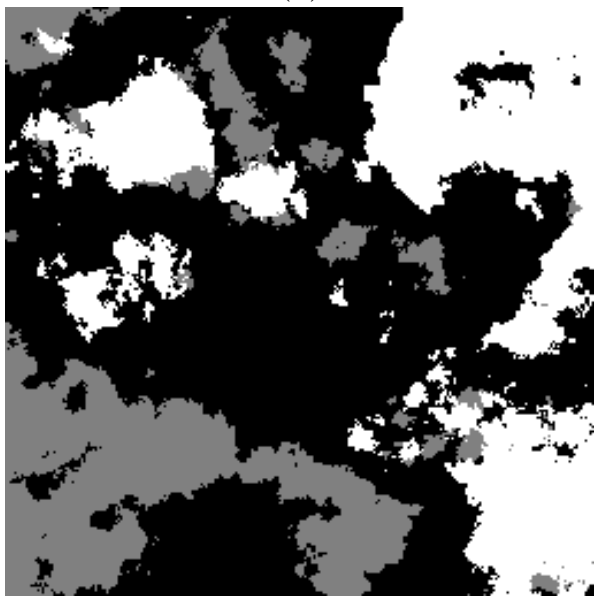

(c)

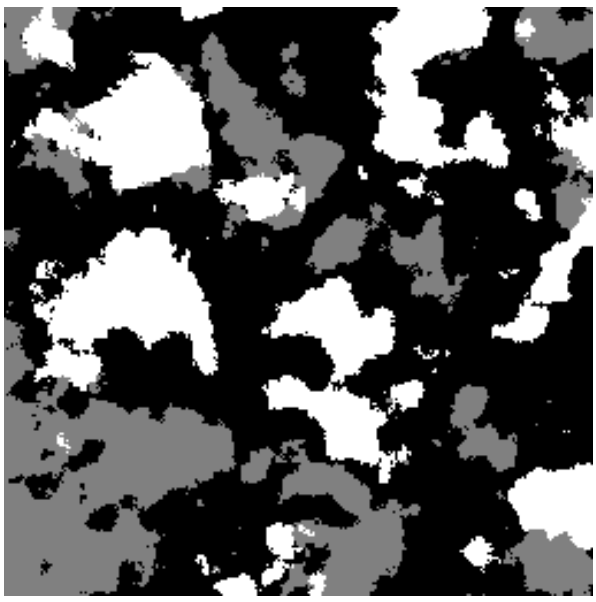

(b)

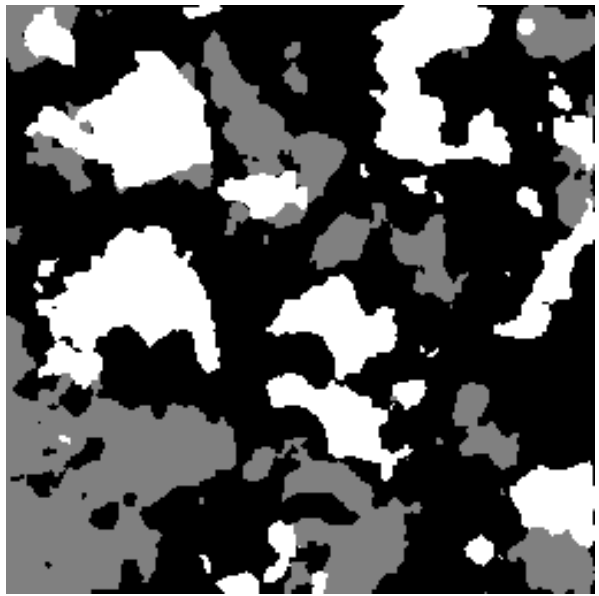

(d)

Figure 11: Sample 29-i: (a) crop of a segmented image, (b) plurigaussian model $M_{4}$, (c) plurigaussian model $M_{5}$ using the Corson model for the covariance functions, (d) plurigaussian model $M_{6}$ with Gaussian low-pass filtering. Note that for the sake of comparison, all images are simulated using fixed Gaussian noises $U_{X, Y}$.

removed using an analytical model for the covariance. This model reads (Corson, 1974):

$$
C(h)=f^{2}+f(1-f) \mathbf{e}^{-c h^{n}},
$$

where $n \leq 1$ and $c$ are two parameters. We follow this procedure and fit the right-hand sides of (31) with the Corson model (32). Values of parameters $n$ and $c$ are given in Tab. 5. These fits are used for the covariances $C_{X, Y}(h)$. Hereafter, this model is referred to as $M_{5}$. Results are represented in Fig. 11c. Noise is not removed using the Corson model, and the method produces sub-optimal models (see Tab. 4, column 5). Indeed, the fit of the covariances using the Corson model is not satisfactory (not shown).

A different approach is followed to remove noise on the contours, that preserves the information provided by the weight functions $w_{X, Y}$. The plurigaussian model $M_{4}$ is filtered using a low-pass normalized-centered Gaussian kernel. This filtering is applied on $Z_{X, Y}$, after the convolution of the Gaussian noise $U_{X, Y}$ by the weight function $w_{X, Y}$. Equivalently, the weight functions $w_{X, Y}^{M_{6}}$ in model $M_{6}$ is replaced by:

$$
w_{X, Y}^{M_{6}}(\mathbf{x})=\left(w_{X, Y}^{M_{4}} * \mathcal{K}\right)(\mathbf{x})
$$

where $\mathcal{K}$ refers to the Gaussian kernel:

$$
\mathcal{K}(\mathbf{x})=\mathbf{e}^{-\|\mathbf{x}\|^{2} / \sigma_{\mathcal{K}}^{2}} .
$$

The value of the variance in the Gaussian kernel is fixed to $\sigma_{\mathcal{K}}=0.055 \mu \mathrm{m}$. This value removes most of the small-length artifacts while preserving the structure of the model. This filtered model is denoted $M_{6}$. As shown 


\begin{tabular}{ccccc}
\hline Sample & $c_{X}$ & $n_{X}$ & $c_{Y}$ & $n_{Y}$ \\
\hline $14-\mathrm{i}$ & 0.138 & 0.859 & 0.159 & 0.863 \\
$14-\mathrm{s}$ & 0.135 & 0.850 & 0.162 & 0.851 \\
$15-\mathrm{i}$ & 0.155 & 0.834 & 0.186 & 0.827 \\
$15-\mathrm{s}$ & 0.153 & 0.813 & 0.168 & 0.844 \\
$24-\mathrm{i}$ & 0.125 & 0.843 & 0.161 & 0.833 \\
$24-\mathrm{s}$ & 0.120 & 0.867 & 0.150 & 0.859 \\
$25-\mathrm{i}$ & 0.145 & 0.785 & 0.175 & 0.821 \\
$25-\mathrm{s}$ & 0.147 & 0.795 & 0.176 & 0.831 \\
$28-\mathrm{i}$ & 0.136 & 0.839 & 0.156 & 0.849 \\
$28-\mathrm{s}$ & 0.206 & 0.798 & 0.228 & 0.813 \\
$29-\mathrm{i}$ & 0.149 & 0.810 & 0.175 & 0.825 \\
$29-\mathrm{s}$ & 0.146 & 0.812 & 0.164 & 0.840 \\
$32-\mathrm{i}$ & 0.108 & 0.864 & 0.108 & 0.893 \\
\hline
\end{tabular}

Table 5: Values of the parameters $(c, n)$ (see Eq. 32) of the Corson model used for each of the 11 samples. For each sample two sets of parameters are identified, the first one $\left(c_{X}, n_{X}\right)$ corresponds to the random set $X$ and the other one $\left(c_{X}, n_{X}\right)$ to the random set $Y$.

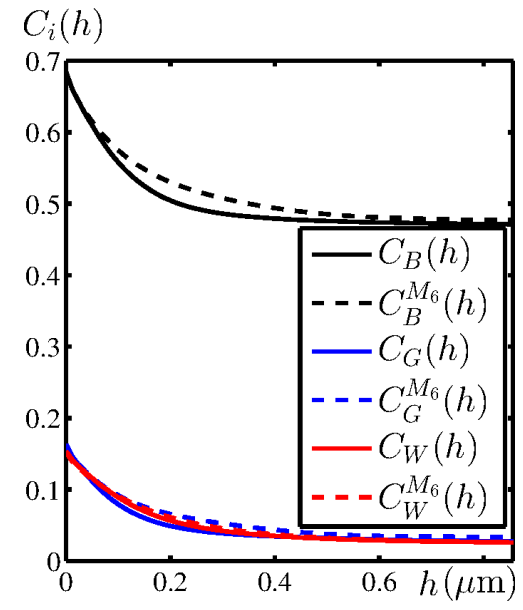

(a)

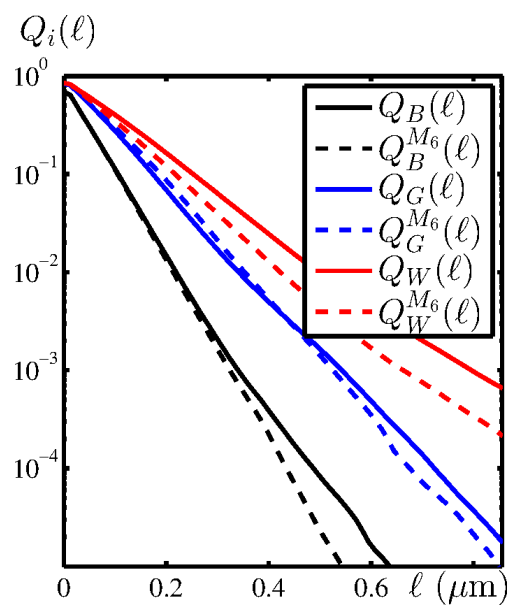

(b)

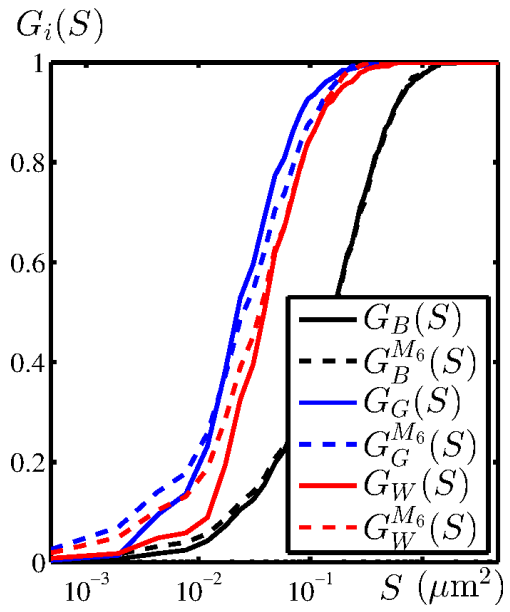

(c)

Figure 12: Sample 29-s: covariances $C_{i}(h)(\mathrm{a})$, linear erosion $Q_{i}(\ell)(\mathrm{b})$ and granulometries $G_{i}(h)$ (c) of the segmented SEM image (solid lines) and that of the optimal Plurigaussian model $M_{6}$ (dotted lines) for phases B (black), G (blue) and $\mathrm{W}$ (red). 


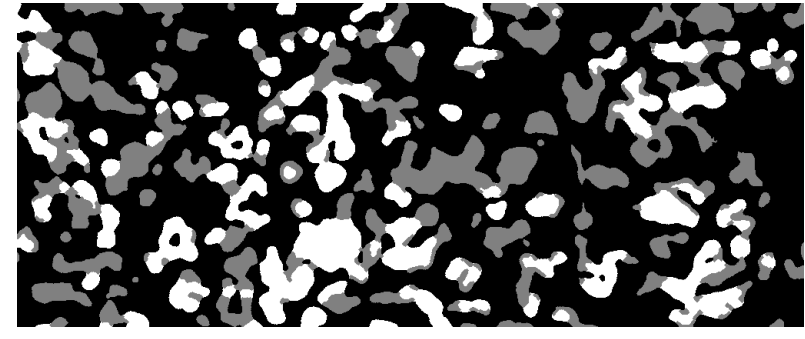

(a)

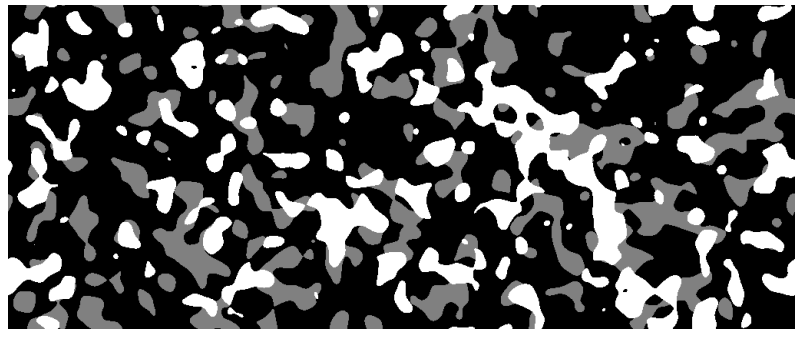

(b)

Figure 13: Crop of a segmented SEM image (a) and 2D section of the corresponding 3D plurigaussian model $M_{6}$ (b) for sample 32 -s.

\begin{tabular}{cccc}
\hline Sample & $f_{W} \pm \epsilon_{f_{W}}$ & $f_{G} \pm \epsilon_{f_{G}}$ & $f_{B} \pm \epsilon_{f_{B}}$ \\
\hline $14-\mathrm{i}$ & $0.149 \pm 0.015$ & $0.197 \pm 0.016$ & $0.654 \pm 0.022$ \\
$14-\mathrm{s}$ & $0.154 \pm 0.012$ & $0.166 \pm 0.011$ & $0.680 \pm 0.015$ \\
$24-\mathrm{i}$ & $0.158 \pm 0.015$ & $0.164 \pm 0.016$ & $0.678 \pm 0.017$ \\
$24-\mathrm{s}$ & $0.181 \pm 0.018$ & $0.198 \pm 0.014$ & $0.620 \pm 0.017$ \\
$25-\mathrm{i}$ & $0.182 \pm 0.019$ & $0.178 \pm 0.011$ & $0.640 \pm 0.019$ \\
$25-\mathrm{s}$ & $0.173 \pm 0.019$ & $0.191 \pm 0.024$ & $0.635 \pm 0.027$ \\
$28-\mathrm{i}$ & $0.184 \pm 0.012$ & $0.193 \pm 0.013$ & $0.623 \pm 0.016$ \\
$28-\mathrm{s}$ & $0.196 \pm 0.014$ & $0.214 \pm 0.014$ & $0.589 \pm 0.017$ \\
$29-\mathrm{i}$ & $0.152 \pm 0.011$ & $0.163 \pm 0.014$ & $0.685 \pm 0.016$ \\
$29-\mathrm{s}$ & $0.190 \pm 0.016$ & $0.201 \pm 0.014$ & $0.608 \pm 0.020$ \\
$32-\mathrm{i}$ & $0.186 \pm 0.021$ & $0.199 \pm 0.015$ & $0.614 \pm 0.020$ \\
\hline
\end{tabular}

Table 6: Volume fractions $f_{i}$ of each phase $i$ estimated on model $M_{6}$ and their associated relative error $\epsilon_{f_{i}}$.

in Fig. (11d) and Tab. (4) (column 6), this filter preserves the statistical properties of the SEM images and the noise is removed.

The volume fraction of each phase in model $M_{6}$ is given in Tab. 6 together with their relative error. The phase volume fractions of model $M_{6}$ (Tab. 6) are close to that measured on the SEM images (Tab. 1). Other morphological measurements on the plurigaussian model $M_{6}$ are represented in Fig. 12 and compared to that of the segmented image. As expected, a very good agreement is found for the two covariance functions $C_{W}$ and $C_{G}$, which are directly used in the plurigaussian model. A good agreement between the plurigaussian model $M_{6}$ and the SEM images is observed as well for the covariance $C_{B}$, and for the linear erosions $Q_{i}$ and granulometry functions $G_{i}$, for the three phases. This result holds even though the linear erosion and granulometry were not used for the identification and the generation of the model. For certain samples, as the one represented in Fig. 12, however, a small misfit is observed for the covariance $C_{B}(h)$. In a few other samples, a small discrepancy appears regarding the granulometry $G_{B}$ (not shown) but overall, the model follows quite closely the measurements. A visual comparison between model $M_{6}$ and the segmented SEM images (Fig. 13) confirms the good behavior of the model. The two images are close to one another.

Exactly the same procedure has been applied to compute $M_{6}$ models corresponding to the DLR and Mines ParisTech samples. The latter are also visually close to the SEM images (Fig. 14). This is especially remarkable as the anode samples have quite different morphologies, due to the particular preparation techniques involved in the fabrication of the cells.

The specific surface areas of the SEM images and $M_{6}$ models are compared in Tab. (7). The contact surface areas between $G$ and $W$, denoted $S_{V_{G W}}$, is computed by estimating the derivative at 0 of the cross-covariance functions of the segmented images and models. The contact surface area between $B$ and $W$, denoted $S_{V_{W B}}$, and between $B$ and $G$, denoted $S_{V_{B G}}$, are estimated in the same way. The comparison is carried out for the ISTEC samples as well as 4 DLR anode samples (labeled $A 1$ to $A 4$ ) and one Mines ParisTech sample (labeled $B 1$ ). The relative error on the measurement of the various surface areas is estimated by computing the derivative of the crosscovariance functions in different ways and studying representativity effects. Details are given in Abdallah (2015). The relative error is estimated to be at most $15 \%$. Overall, good agreement is found between the models and SEM images, except for the contact surface area between $G$ and $W$ for the DLR samples, which are underestimated by model $M_{6}$ by a factor 2 . Use of the parameter $\sigma_{\mathcal{K}}$ in (33) would presumably provide additional degrees of freedom for improving the model in this respect, as will be seen hereafter. 


\begin{tabular}{ccccccc}
\hline & \multicolumn{2}{c}{$S_{V_{G W}}$} & \multicolumn{2}{c}{$S_{V_{W B}}$} & \multicolumn{2}{c}{$S_{V_{G B}}$} \\
\cline { 2 - 7 } Sample & $M_{6}$ & SEM & $M_{6}$ & SEM & $M_{6}$ & SEM \\
\hline $14-\mathrm{i}$ & 1.36 & 1.23 & 4.20 & 4.68 & 7.90 & 7.97 \\
$14-\mathrm{s}$ & 1.02 & 1.07 & 4.43 & 4.88 & 6.99 & 6.93 \\
$15-\mathrm{i}$ & 0.96 & 1.04 & 3.74 & 4.22 & 5.46 & 7.34 \\
$15-\mathrm{s}$ & 1.42 & 1.47 & 3.91 & 4.80 & 6.34 & 8.43 \\
$24-\mathrm{i}$ & 1.10 & 1.09 & 4.56 & 4.32 & 5.81 & 6.10 \\
$24-\mathrm{s}$ & 1.41 & 1.42 & 4.47 & 4.74 & 6.75 & 6.99 \\
$25-\mathrm{i}$ & 1.36 & 1.18 & 4.97 & 5.24 & 6.57 & 7.15 \\
$25-\mathrm{s}$ & 1.46 & 1.23 & 4.83 & 5.31 & 7.10 & 7.78 \\
$28-\mathrm{i}$ & 1.59 & 1.20 & 4.95 & 5.45 & 6.89 & 7.41 \\
$28-\mathrm{s}$ & 1.84 & 1.42 & 5.05 & 8.20 & 7.30 & 10.55 \\
$29-\mathrm{i}$ & 1.13 & 0.90 & 4.73 & 5.11 & 6.26 & 6.80 \\
$29-\mathrm{s}$ & 1.70 & 1.33 & 4.99 & 5.61 & 7.27 & 7.89 \\
$32-\mathrm{i}$ & 1.43 & 1.47 & 4.38 & 4.08 & 5.32 & 5.37 \\
$32-\mathrm{s}$ & 1.59 & 1.78 & 4.32 & 4.14 & 5.64 & 5.89 \\
$33-\mathrm{i}$ & 0.99 & 1.14 & 3.94 & 3.88 & 5.36 & 5.11 \\
$33-\mathrm{s}$ & 1.31 & 1.38 & 4.33 & 4.14 & 5.62 & 5.22 \\
A1-i & 0.56 & 1.42 & 3.00 & 2.25 & 4.99 & 4.69 \\
A1-s & 0.54 & 1.21 & 2.81 & 2.32 & 4.69 & 4.56 \\
A2-i & 0.93 & 1.79 & 3.77 & 3.47 & 5.57 & 4.84 \\
A2-s & 1.26 & 2.22 & 4.13 & 3.74 & 5.90 & 4.76 \\
A3-i & 0.98 & 1.74 & 3.88 & 3.60 & 5.19 & 4.92 \\
A3-s & 1.23 & 2.05 & 4.20 & 4.18 & 5.27 & 4.78 \\
A4-i & 0.71 & 1.07 & 3.64 & 3.55 & 5.05 & 4.70 \\
A4-s & 1.05 & 1.16 & 4.02 & 4.22 & 5.40 & 5.20 \\
B1-i & 3.85 & 3.96 & 5.28 & 5.78 & 7.90 & 8.00 \\
B1-s & 4.06 & 4.59 & 5.46 & 5.78 & 7.67 & 7.19 \\
\hline & & & & & &
\end{tabular}

Table 7: Specific surface areas $S_{V_{G W}}, S_{V_{W B}}$ and $S_{V_{G B}}\left(\right.$ in $\mu \mathrm{m}^{-1}$ ) between any two phases measured on SEM images and on the corresponding $M_{6}$ model. Discrepancies between the models and SEM images are highlighted in dark and light gray when the model underestimates and overestimates, respectively, the measurement carried out on the SEM image. 


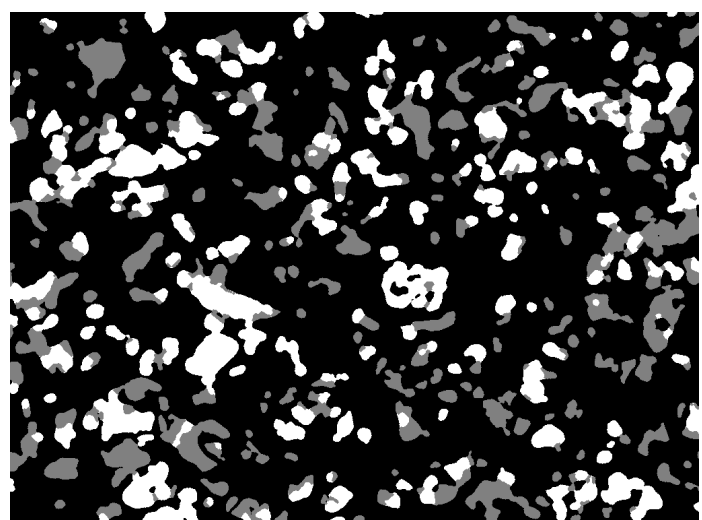

(a)

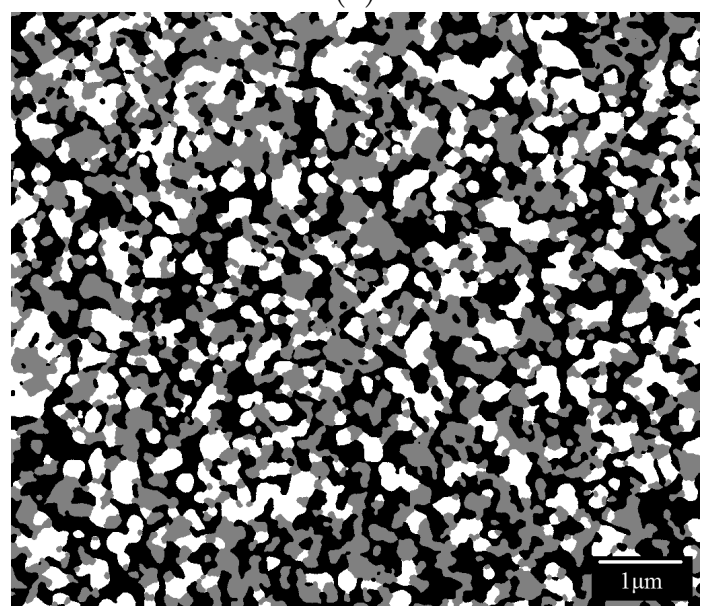

(c)

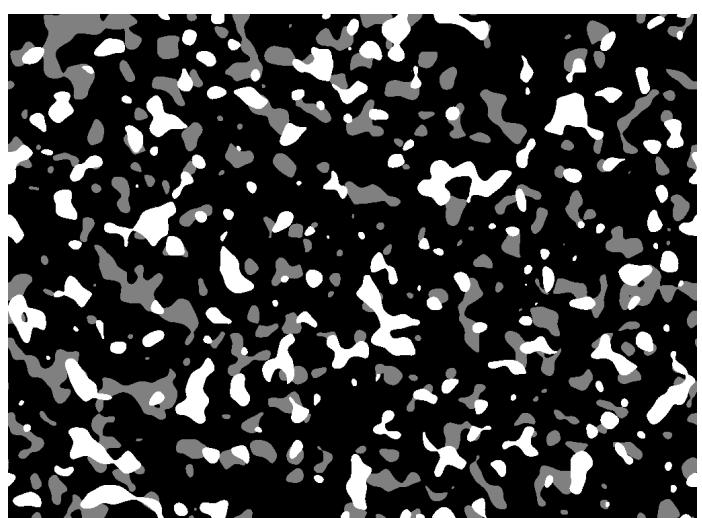

(b)

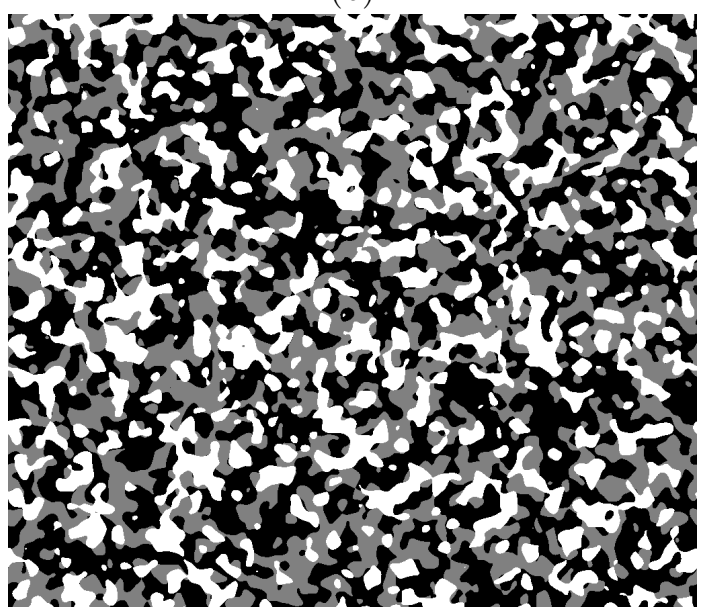

(d)

Figure 14: SEM images (a, c) vs. microstructure model $M_{6}(\mathrm{~b}, \mathrm{~d})$ for samples A4-i (a, b) and B1-i (c, d). 


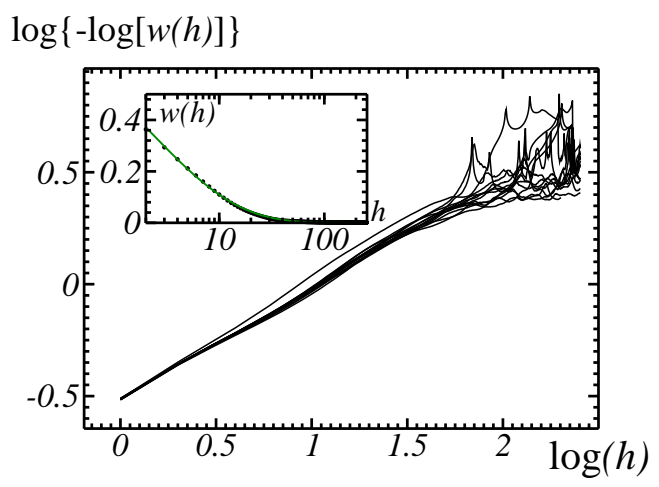

Figure 15: Quantity $\log [-\log w(h)]$ vs. $\log h$, computed on a set of 12 SEM images of anode layers. Top-left quadrant: weight function $w_{X}(h)$ determined from $(29),(30)$ and $(31)$, for the anode layer 29-s (dots); fit $w_{X}(h)=\mathrm{e}^{-\sqrt{h / 2.0}}$ (solid line). with parameter $\alpha_{X}=2.0$ pixels $\approx 25 \mathrm{~nm}$.

The model $M_{6}$ is based on the covariance functions of $X$ and $Y$, or equivalently, of $B, W$ and $G$. Thus a parametrization of the latter functions is needed to obtain an analytical model for $M_{6}$. Numerical investigations show that the weight functions $w_{X, Y}(h)$ for $X$ and $Y$ are close to the law:

$$
w_{X, Y}(h)=\mathrm{e}^{-\left(h / \alpha_{X, Y}\right)^{\beta} X, Y},
$$

where, depending on the material, $0.5 \leq \beta_{X} \leq 0.55$ and $2.0 \leq \alpha_{X} \leq 2.07$. The two parameters are not independent. Because of the condition (28), they satisfy:

$$
\alpha_{X, Y}=2^{1 / \beta_{X, Y}}\left[\Gamma\left(1+\frac{1}{\beta_{X, Y}}\right)\right]^{-1},
$$

where $\Gamma$ is the extended factorial function. A numerical fit of $w_{X}(h)$ is illustrated in Fig. (15). In this figure, the quantity $\log \left[-\log w_{X}(h)\right]$ is plotted as a function of $\log h$. The form (35) leads to the expansions, for $h \rightarrow 0$ :

$$
\rho_{X, Y}(h) \approx 1-\frac{h}{2}, \quad C_{X, Y}(h) \approx C_{X, Y}(0)-\frac{\mathrm{e}^{-\lambda_{X, Y}^{2} / 2}}{2 \pi} \sqrt{h} .
$$

The expansion for $C_{X, Y}(h)$ with first-order correction $\sim \sqrt{h}$ indicates an infinite specific surface area, which explains the roughness of the contours observed for model $M_{4}$ (Fig. 11b). For model $M_{6}$, as a result of the regularization (33) by a Gaussian kernel, one finds instead, for $h \rightarrow 0$ :

$$
\rho_{X, Y}(h) \approx 1-\frac{h^{2}}{4 \sigma^{2}}, \quad C_{X, Y}(h) \sim C(0)-\frac{h}{2 \sigma},
$$

so that smooth boundaries and a finite surface area are obtained for the random set. In that case, the specific surface areas of $X$ and $Y$ is given by the parameters $\sigma_{\mathcal{K}}$ (Eq. 34) and $\lambda_{X, Y}$ as:

$$
S_{V_{X}}=\frac{1}{\sigma_{\mathcal{K}} \pi} e^{-\lambda_{X}^{2} / 2}, \quad S_{V_{Y}}=\frac{1}{\sigma_{\mathcal{K}} \pi} e^{-\lambda_{Y}^{2} / 2} .
$$

The contact surface areas between any two phases are given by:

$$
S_{V_{W G}}=f_{Y} S_{X}, \quad S_{V_{W B}}=\left(1-f_{Y}\right) S_{X}, \quad S_{V_{B G}}=\left(1-f_{X}\right) S_{Y} .
$$

The formula above match the numerical measures for the contact areas in the the model (Tab. 7).

\section{Conclusion}

A methodology has been presented for simulating 3D models of three-phase anode composites using 2D SEM images. Such images should first be segmented, which requires removing the "halo" (or 3D) effect present in porous materials. For moderate halo effects, a combination of dilation and openings is sufficient. Second, the segmented microstructures have been characterized by a set of stereological measurements, in particular the covariance and cross-covariance functions. 
Third, a generic three-phase random model has been introduced, based on two underlying independent random sets. We showed that this generic model results in simple constraints on the covariance and cross-covariance functions that can be tested in practice. For the anode layers studied here, the covariance and cross-covariance functions were found to be roughly compatible with one of the possible combinations of the three-phase model. In a fourth step, several models, namely Boolean sets and plurigaussian fields, were explored for the underlying random sets defining the three-phase model. Good agreement was obtained with plurigaussian models in terms of covariance, granulometry and linear erosion functions and the plurigaussian models were found to be visually very close to the materials. Furthermore, the models can be simulated very easily and efficiently, using the covariance function of the material or an analytical model that we propose. Similar results were obtained on anode layers of different origins and microstructures, highlighting the versatility of the proposed method.

We emphasize that the methodology developed in this work is applicable to materials with an arbitrary number of phases. In the general case, an $n$-phases microstructure is modeled using $n-1$ underlying independent random sets. The independency assumption enforce a set of constraints on the covariance and cross-covariance functions of the material's phases, as studied in (Jeulin, 1979). This work demonstrates that such $n$-phases models are useful for simulating real materials.

Acknowledgements The research leading to the results presented has received funding from the European Union Seventh Framework Programme (FP7/ 2007-2013) for the Fuel Cells and Hydrogen Joint Technology Initiative under grant agreement 303429. The authors are grateful to Alessandra Sanson, Elisa Mercadelli and Angela Gondoli for providing the ISTEC anode samples and to David Masson, Anthony Chesnaud and Alain Thorel for image acquisitions and Armines anode samples. The authors also thank Rémi Costa, Feng Han, Vitaly Yurkiv and Aitor Hornes for providing the DLR anode samples. Finally, the authors are grateful to two anonymous reviewers for helpful suggestions.

\section{References}

Abdallah, B. (2015). Analyse morphologique et modélisation pour l'optimisation structurelle d'éléctrodes. PhD Thesis, Mines ParisTech (in preparation).

Abdallah, B., Willot, F., and Jeulin, D. (2015). Stokes flow through a Boolean model of spheres: Representative volume element, Transp. Por. Media 109, 711-726.

Armstrong, M., Galli, A., Beucher, H., Loc'h, G., Renard, D., Doligez, B., Eschard, R., and Geffroy, F. Plurigaussian Simulations in Geosciences. Springer, Berlin, Heidelberg, 2011.

Badwal, S.P.S., Giddey, S., Kulkarni, A., and Munnings, C. (2014). Review of progress in high temperature solid oxide fuel cells. J. Aust. Cer. Soc. 50, 23-37.

Bron, F. and Jeulin, D. (2004). Modelling a food microstructure by random sets. Image Analysis ES Stereology 23, 33-44.

Corson, P.B. (1974). Correlation functions for predicting properties of heterogeneous materials. I. Experimental measurement of spatial correlation functions in multiphase solids. J. Appl. Physics 45, 3159-3164.

Gillibert, L., Peyrega, C. Jeulin, D., Guipont, V., and Jeandin, M. (2012). 3D multiscale segmentation and morphological analysis of X-ray microtomography from cold-sprayed coatings. Journal of Microscopy 248, 187199.

Gostovic, D., Smith, J.R., Kundinger, D.P., Jones, K.S., and Wachsman, E.D. (2007). Three-Dimensional Reconstruction of Porous LSCF Cathodes. Electrochemical and Solid-State Letters 10, B214.

Greco, A., Jeulin, D. and Serra, J. (1979). The use of the texture analyser to study sinter structure: application to the morphology of calcium ferrites encountered in basic sinters of rich iron ores. Journal of Microscopy 116, 199-211.

Grove, W. R. (1839). On voltaic series and the combination of gases by platinum. The London and Edinburgh Philosophical Magazine and Journal of Science 14, 127-130.

Iwai, H., Shikazono, N., Matsui, T., Teshima, H., Kishimoto, M., Kishida, R., Hayashi, D., Matsuzaki, K., Kanno, D., Saito, M., Muroyama, H,. Eguchi, K., Kasagi, N., and Yoshida, H. (2010). Quantification of SOFC anode microstructure based on dual beam FIB-SEM technique. Journal of Power Sources 195, 955-961.

Jeulin, D. (2014). Modelling three phase random media. Internal report, CMM, Mines ParisTech N-01/14MM. 
Jeulin, D. (1979). Morphologie Mathématique et Propriétés Physiques des agglomérés de minerais de fer et du coke métallurgique, PhD Thesis, École des Mines de Paris.

Joos, J., Carraro, T., Weber, A., and Ivers-Tiffée, E. (2011). Reconstruction of porous electrodes by FIB/SEM for detailed microstructure modeling. Journal of Power Sources 196, 7302-7307.

Joos, J., Ender, M., Carraro, T., Weber, A., and Ivers-Tiffée, E. (2012). Representative volume element size for accurate solid oxide fuel cell cathode reconstructions from focused ion beam tomography data. Electrochimica Acta 82, 268-276.

Kanit, T., Forest, S., Galliet, I., Mounoury, V. and Jeulin, D. (2003). Determination of the size of the representative volume element for random composites: statistical and numerical approach. International Journal of solids and Structures 40, 3647-3679.

Laguna-Bercero, M.A. (2012). Recent advances in high temperature electrolysis using solid oxide fuel cells: A review. Journal of Power Sources 203, 4-16.

Lantuéjoul, C. Geostatistical Simulation - Models and algorithm. Springer, Berlin, Heidelberg, pp. 183-240, 2002.

Lanzini, A., Leone, P., and Asinari, P. (2009). Microstructural characterization of solid oxide fuel cell electrodes by image analysis technique. Journal of Power Sources 194, 408-422.

Masson, D., Abdallah, B., Willot, F., Jeulin, D., Mercadelli, E., Sanson, A., Chesnaud, A., and Thorel, A. (2015). Morphological modeling of a metal foam SOFC configuration. ECS Trans. 68, 2951-2960.

Matheron, G. Élements pour une théorie des milieux poreux. Masson, Paris, 1967.

Matheron, G. The theory of regionalized variables and its applications. Paris School of Mines publications, Paris, 1971.

Nelder, J.A. and Mead, r. (1965). A Simplex Method for Function Minimization. The Computer Journal 7, 308-313.

Shearing, P.R., Golbert, J., Chater, R.J., and Brandon, N.P. (2009). 3D reconstruction of SOFC anodes using a focused ion beam lift-out technique. Chemical Engineering Science 64, 3928-3933.

Tomasi, C. and Manduchi, R. (1998). Bilateral Filtering for Gray and Color Images. In Proceedings of the 1998 IEE International Conference on Computer Vision, pp. 839-846, Bombay, India.

Willot, F. (2015). Mean covariogram of a cylinder and applications to Boolean random sets. Journal of contemporary mathematical analysis. In press.

Wilson, J.R., Kobsiriphat, W., Mendoza, R., Chen, H.Y., Hiller, J.M., Miller, D.J., Thornton, K., Voorhees, P.W , Adler, S.B., and Barnett, S.A. (2006). Three-dimensional reconstruction of a solid-oxide fuel-cell anode. Nature materials 5, 541-544.

Yurkiv, V., Constantin, G., Hornes, A., Gondolini, A., Mercadelli, E., Sanson, A., Dessemond, L., and Costa, R. (2015). Towards understanding surface chemistry and electrochemistry of $\mathrm{La}_{0.1} \mathrm{Sr}_{0.9} \mathrm{TiO}_{3-\alpha}$ based solid oxide fuel cell anodes. Journal of Power Sources 287, 58-67.

Zhang, S., Lynch, M., Gokhale, A.M., and Liu, M. (2009). Unbiased characterization of three-phase microstructure of porous lanthanum doped strontium manganite/yttria-stabilized zirconia composite cathodes for solid oxide fuel cells using atomic force microscopy and stereology. Journal of Power Sources 192, 367-371. 